\title{
Comparing Multimodal Pilot Pitch Control Behavior Between Simulated and Real Flight
}

\author{
P.M.T. Zaal, ${ }^{*}$ D.M. Pool ${ }^{\dagger}$ \\ M.M. van Paassen, ${ }^{\ddagger}$ and M. Mulder ${ }^{\S}$ \\ Delft University of Technology, Delft, The Netherlands
}

\begin{abstract}
In order to improve the tuning process of flight simulator motion cueing filters and support the development of objective simulator motion cueing requirements, a better understanding of how multimodal pilot control behavior is affected by simulator motion fidelity is required. To this end, an experiment was performed where seven pilots performed a pitch target-following disturbance-rejection task in a simulator under four different motion cueing settings, in addition to performing the task in a real aircraft, which served as the baseline condition. Differences between the simulator and aircraft experiment setup were minimized. Small remaining differences in the display and the sidestick setup slightly affected the experiment dependent measures. However, the effects introduced by the motion cueing settings were far more apparent. When motion fidelity was increased to full aircraft motion, pilots were able to increase performance in attenuating the disturbance signal significantly. In addition, for increased motion fidelity, a change in multimodal pilot control behavior was observed by a decrease in pilot visual lead, while visual and vestibular perception delays increased. Pilot performance and control behavior in the simulator condition with full pitch motion and filtered pitch and c.g. heave motion was most similar to the in-flight condition.
\end{abstract}

\section{Nomenclature}

$\begin{array}{llll}A_{d, t} & \text { sinusoid amplitude, } \mathrm{V}, \mathrm{deg} & H_{p \theta} & \text { pilot pitch response } \\ a_{z} & \text { pilot station acceleration, } \mathrm{m} \mathrm{s}^{-2} & H_{\theta, \delta_{e}} & \text { aircraft pitch dynamics } \\ a_{z_{c . g}} & \text { c.g. acceleration, } \mathrm{m} \mathrm{s}^{-2} & j & \text { imaginary unit } \\ a_{z_{\theta}} & \text { pitch acceleration, } \mathrm{m} \mathrm{s}^{-2} & K_{h, \delta_{e}} & \text { c.g. heave dynamics gain } \\ e & \text { tracking error signal, deg } & K_{m} & \text { pilot motion gain } \\ f_{d} & \text { disturbance forcing } & K_{m f} & \text { motion filter gain } \\ & \text { function, V } & K_{s} & \text { stick gain } \\ f_{t} & \text { target forcing function, deg } & K_{v} & \text { pilot visual gain } \\ H_{a_{z_{c g}}, \delta_{e}} & \text { aircraft heave dynamics } & K_{\theta, \delta_{e}} & \text { pitch dynamics gain } \\ H_{c} & \text { controlled dynamics } & k & \text { sinusoid index } \\ H_{f b w} & \text { fly-by-wire dynamics } & l & \text { distance between aircraft } \\ H_{m f} & \text { motion filter } & & \text { c.g. and pilot station, } \mathrm{m} \\ H_{o l} & \text { open-loop dynamics } & n & \text { pilot remnant signal, deg } \\ H_{p a_{z}} & \text { pilot heave response } & n_{d, t} & \text { forcing function frequency } \\ H_{p e} & \text { pilot visual response } & & \text { integer factor } \\ H_{p p} & \text { prepositioning filter } & s & \text { Laplace variable }\end{array}$

*Ph.D. student, Control and Simulation Division, Faculty of Aerospace Engineering, P.O. Box 5058, 2600GB Delft, The Netherlands; p.m.t.zaal@tudelft.nl. Student member AIAA.

${ }^{\dagger}$ Ph.D. student, Control and Simulation Division, Faculty of Aerospace Engineering, P.O. Box 5058, 2600GB Delft, The Netherlands; d.m.pool@tudelft.nl. Student member AIAA.

${ }^{\ddagger}$ Associate Professor, Control and Simulation Division, Faculty of Aerospace Engineering, P.O. Box 5058, 2600GB Delft, The Netherlands; m.m.vanpaassen@tudelft.nl. Member AIAA.

$\S$ Professor, Control and Simulation Division, Faculty of Aerospace Engineering, P.O. Box 5058, 2600GB Delft, The Netherlands; m.mulder@tudelft.nl. Senior Member AIAA. 


\begin{tabular}{|c|c|c|c|}
\hline$T_{A_{1}, A_{2}}$ & amplitude filter & $\tau_{v}$ & pilot visual delay, $\mathrm{s}$ \\
\hline & $\begin{array}{l}\text { time constants, s } \\
\text { c.g. heave dynamics }\end{array}$ & $\omega$ & $\begin{array}{l}\text { frequency, rad } \mathrm{s} \\
\text { motion filter break frequency, } \mathrm{rad}^{-1}\end{array}$ \\
\hline$I_{2}, h_{3}$ & time constants, s & $\begin{array}{l}\omega_{b_{m f}} \\
\omega_{b_{p p}}\end{array}$ & prepositioning filter \\
\hline$T_{l e a d}$ & pilot lead time constant, $\mathrm{s}$ & & break frequency, $\operatorname{rad~s}^{-1}$ \\
\hline$T_{l a g}$ & pilot lag time constant, s & $\omega_{c}$ & crossover frequency, $\mathrm{rad} \mathrm{s}^{-1}$ \\
\hline$T_{m}$ & measurement time, $\mathrm{s}$ & $\omega_{d, t}$ & sinusoid frequency, $\mathrm{rad} \mathrm{s}^{-1}$ \\
\hline $\begin{array}{l}T_{\theta_{2}} \\
t\end{array}$ & $\begin{array}{l}\text { pitch dynamics time constant, } \mathrm{s} \\
\text { time, s }\end{array}$ & $\omega_{m}$ & $\begin{array}{l}\text { measurement time } \\
\text { break frequency, } \operatorname{rad~s}^{-1}\end{array}$ \\
\hline$u_{y}$ & pilot control signal, $\mathrm{V}$ & $\omega_{n m}$ & neuromuscular frequency, $\operatorname{rad~s}^{-1}$ \\
\hline Symbols & & $\omega_{n_{m f}}$ & $\begin{array}{l}\text { motion filter } \\
\text { natural frequency, } \operatorname{rad~s}^{-1}\end{array}$ \\
\hline $\begin{array}{l}\delta_{e} \\
\theta\end{array}$ & $\begin{array}{l}\text { elevator deflection, deg } \\
\text { pitch angle, deg }\end{array}$ & $\omega_{n_{p p}}$ & $\begin{array}{l}\text { prepositioning filter } \\
\text { natural frequency, } \operatorname{rad~s}^{-1}\end{array}$ \\
\hline$\sigma^{2}$ & variance & $\omega_{s p}$ & short-period frequency, $\mathrm{rad} \mathrm{s}^{-1}$ \\
\hline$\varphi_{m}$ & phase margin, deg & $\zeta_{m f}$ & neuromuscular damping ratio \\
\hline$\phi_{d, t}$ & sinusoid phase shift, rad & $\zeta_{n m}$ & motion filter damping ratio \\
\hline & display time delay, s & & $\begin{array}{l}\text { prepositioning filter damping ratio } \\
\text { short-period dampino ratio }\end{array}$ \\
\hline & motion delay, s & & 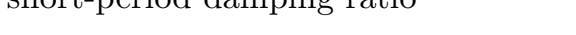 \\
\hline
\end{tabular}

\section{Introduction}

In order for a high fidelity flight simulator to be an effective tool for research and training, a pilot performing a task in this simulator should behave as in the real aircraft. ${ }^{1}$ However, it has been shown that for skill-based aircraft control tasks, pilot performance and control behavior are significantly affected by simulator motion cueing settings. ${ }^{2-5}$ Current technology-centered fidelity metrics do not reflect to what extent a simulator is able to induce real-flight pilot behavior, as they do not incorporate knowledge about human perception and control processes. This warrants the development of a new fidelity metric that determines the simulator's ability to induce real-flight pilot control behavior. ${ }^{6}$

At the Faculty of Aerospace Engineering of Delft University of Technology (TU Delft) a research project is dedicated to developing such a behavioral fidelity metric. ${ }^{7}$ This paper focuses on an important step in this project, that is, to determine how pilot control behavior is affected by the limited physical motion stimuli that are typically provided in a simulator. This is accomplished by identifying pilot control behavior in a simulator under different motion cueing conditions, and comparing this behavior to the baseline control behavior determined in real flight. In the next steps of the project, this knowledge will be used to trace behavioral discrepancies back to the way motion stimuli are presented in the simulator. Standards and metrics for behavioral fidelity will be developed, and, finally, motion cueing algorithms can be optimized to increase simulator behavioral fidelity based on objectively-defined targets.

Pilot control behavior can be characterized by estimating the parameter values of quasi-linear pilot models. In previous studies, this approach was used to compare pilot control behavior between real and simulated flight. $^{8-14}$ However, in all these studies only a single pilot response function was identified, without distinguishing between the contributions of different perceptual modalities, for example, visual and vestibular. In a multi-sensory environment, such as a motion-base flight simulator, this may obscure the pilot's ability to adopt a different control strategy by a different use of perceptual modalities. Therefore, to compare control behavior between real and simulated flight adequately, multimodal pilot models need to be identified that are able to distinguish the pilot's use of his modalities separately. The identification of these models requires a combined target-following disturbance-rejection task, as multiple forcing functions need to be inserted at different locations in the control loop, to allow for accurate estimation of the model parameters. ${ }^{3,15,16}$

This paper presents the results of a study in which, for the first time, multimodal pilot control behavior in a pitch attitude control task is compared between real and simulated flight. To facilitate the targetfollowing disturbance-rejection task in real flight, an experimental fly-by-wire (FBW) system was developed and installed into the Cessna Citation II laboratory aircraft operated by the TU Delft. ${ }^{17}$ Using this FBW system a disturbance forcing function was added to the pilot control signal to allow for a deterministic and 


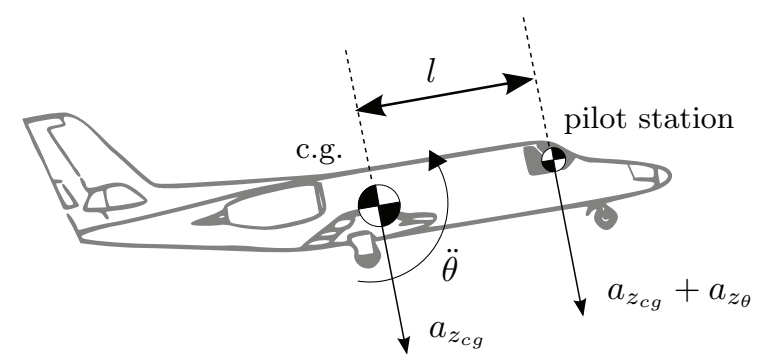

Figure 1. Aircraft motion at the center of gravity and pilot station during a pitch maneuver.

independent disturbance of the aircraft motion, while a target forcing function was visualized on a visual display in the cockpit. In addition to performing the task in the aircraft, pilots performed the task in the SIMONA Research Simulator (SRS) at TU Delft under four different motion cueing conditions, to investigate the effect of limited simulator motion fidelity on pilot performance and multimodal control behavior.

In Section II, the pitch attitude control task for the identification of multimodal pilot control behavior in real and simulated flight will be discussed. Next, in Section III, the experiment setup will be given for both the simulator and the in-flight measurements. This is followed by a comparison of pilot performance and multimodal pilot model identification results from the aircraft and simulator data in Section IV. The paper ends with a discussion and conclusions.

\section{Pitch Attitude Control Task}

In order to compare multimodal pilot control behavior between real and simulated flight, a pitch attitude tracking task was performed in both the Cessna Citation II laboratory aircraft and the SRS. This section describes the pitch tracking task, the controlled aircraft dynamics, and the pilot model used to quantify multimodal pilot control behavior.

\section{II.A. Pitch and Heave Motion Cues}

As visualized in Figure 1, in a Cessna Citation II - as in most conventional fixed-wing aircraft - pilots are seated a considerable distance in front of the center of gravity (c.g.). When performing a pitch attitude control task, pilots are subject to both rotational pitch and linear heave motion. The rotational pitch motion $\ddot{\theta}$ is a direct result of the change in aircraft pitch attitude. The total linear heave motion at the pilot station is a combination of pitch heave $a_{z_{\theta}}$ and c.g. heave motion $a_{z_{c g}}{ }^{4,5}$ Pitch heave is the linear acceleration induced by the pitch rotation of the aircraft and the relative position of the pilot station in front of the c.g.. Center of gravity heave results from relatively slow changes in aerodynamic lift due to the change in aircraft angle of attack while pitching. Figure 1 shows that the total heave at the pilot station can be described by:

$$
a_{z}=a_{z_{c g}}+a_{z_{\theta}}=a_{z_{c g}}-l \ddot{\theta}
$$

where $l$ is the distance between the aircraft c.g. and the pilot station. For the Cessna Citation II, this distance is approximately $3.2 \mathrm{~m}$.

Pitch rotational motion and both types of heave motion are directly correlated with the change in aircraft pitch attitude and, when perceived by pilots, allows them to close additional feedback loops around the controlled aircraft dynamics, allowing for an increase in performance. ${ }^{18}$ In conventional flight simulators, linear accelerations resulting from the aircraft model need to be severely attenuated to keep the simulator cab within the limits of the motion system. Classical linear washout filters are most commonly used for attenuation in magnitude and phase. ${ }^{19}$ For the pitch control task considered in this paper, and for most flight simulation applications in general, the c.g. heave component is the most problematic for motion cueing due to its low-frequency but high-amplitude characteristics.

In preparation to the experiment described in the current paper, two experiments have been performed in the SRS to investigate the effects of limited motion cues on pilot performance and control behavior in a pitch attitude control task. In the first experiment, described in Ref. 4, the effects of rotational pitch and the two heave motion components have been investigated. Ref. 5 describes the second experiment in 


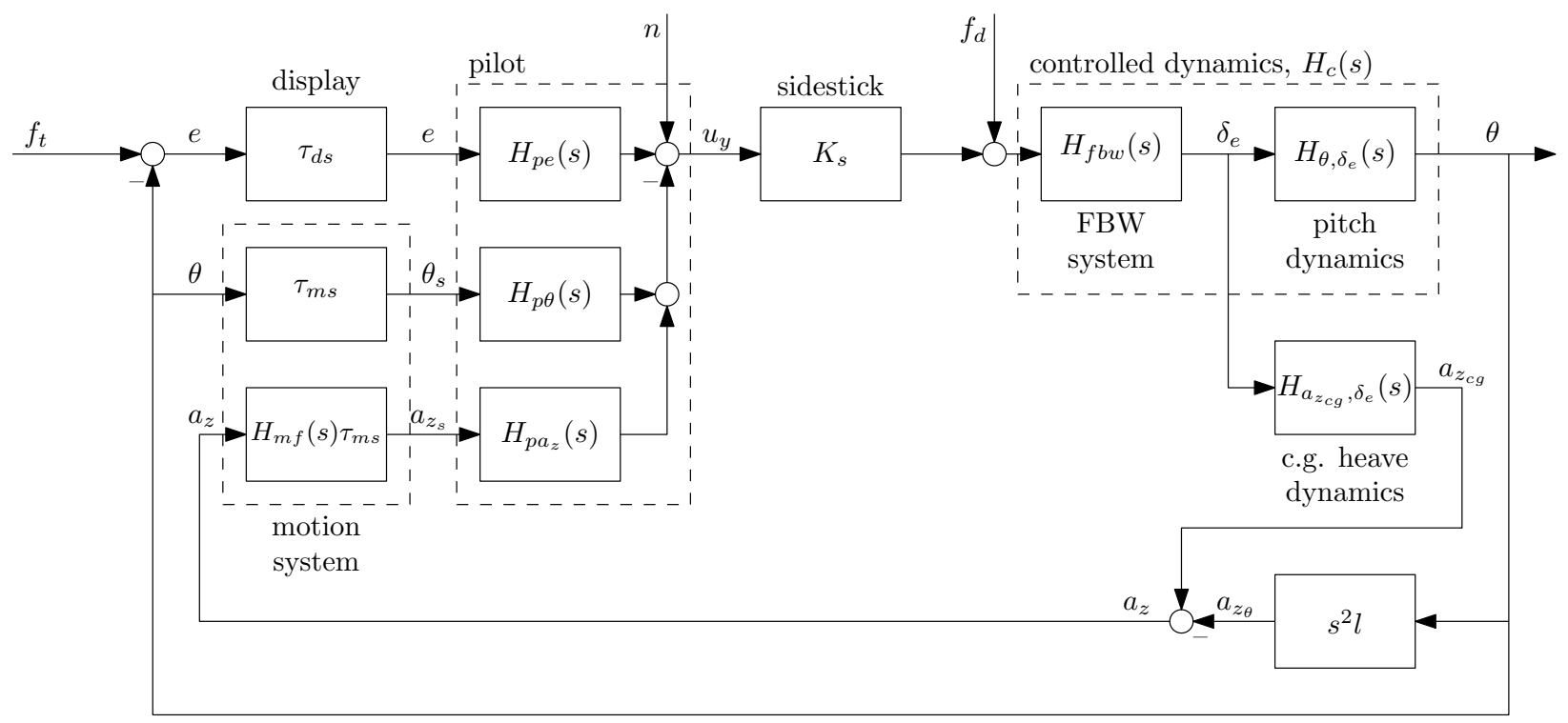

Figure 2. Simulator closed-loop control task.

which the effects of different heave motion filter settings were investigated. These experiments have been performed using a model of the longitudinal dynamics of a Cessna Citation I, which are very similar to those of a Citation II. ${ }^{13}$

The experiments showed that multimodal pilot control behavior is significantly affected by the different motion components and the level of fidelity with which they are presented to the pilot. Rotational pitch motion and pitch heave motion significantly improved tracking performance. When pitch heave motion was presented one-to-one, the effects on pilot performance and control behavior were found to be very similar to the effects of rotational pitch motion. Pilot performance was found to be significantly reduced, however, when the pitch heave motion was attenuated with a gain or filtered using a linear washout filter, that is, the fidelity of the motion was reduced. The c.g. heave motion was found to have no significant effect on pilot performance at all. However, a significantly higher visual lead was observed when c.g. heave was present, indicating a decreased use of the total simulator motion to improve performance.

It was not possible to represent c.g. heave one-to-one in these experiments, as this would drive the SRS motion system beyond its physical limits. Hence, there was no baseline condition with full aircraft motion to investigate truly the effects of attenuating the motion in a simulator. The experiment described in this current paper was designed to compare pilot performance and multimodal control behavior in different simulator motion conditions to an in-flight baseline condition, that is, one-to-one pitch and full heave motion. The experimental conditions in this experiment have been kept as similar as possible to the previous experiments described in Refs. 4 and 5 to allow for a direct comparison of the results.

\section{II.B. Closed-Loop Control Task}

Figure 2 shows the compensatory closed-loop target-following disturbancerejection task used in this study. This type of control task has been considered in many previous investigations into multimodal human manual control behavior ${ }^{3,4,20}$ and techniques for the identification of control behavior in such tasks are well established. ${ }^{15,21,22}$ The figure shows a pilot performing the task in a motion-base simulator.

In this compensatory tracking task, the objective of the pilot is to minimize actively the deviation of the aircraft pitch attitude $\theta$ from a desired pitch attitude or target forcing function $f_{t}$. In addition, the pilot needs to minimize a disturbance acting on the aircraft, induced by a dis-

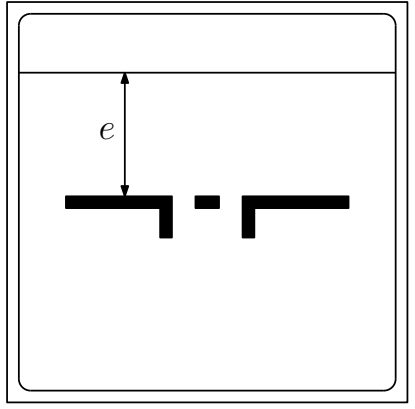

Figure 3. Compensatory display. turbance forcing function $f_{d}$. The deviation from the target forcing function is visualized by the error $e$ on the compensatory display depicted in Figure 3. Note that - as opposed to a pursuit display - the error is 
the only task variable available on the visual display, that is, there is no visual information on the actual pitch attitude of the aircraft.

The pilot controls the aircraft dynamics using a sidestick with a gain $K_{s}$. The summation of the disturbance forcing function $f_{d}$ and the stick output serves as the control input for a model of the aircraft's FBW system $H_{f b w}(s)$. This model provides the aircraft dynamics with elevator control inputs $\delta_{e}$. The aircraft pitch and c.g. heave responses are given by the transfer functions $H_{\theta, \delta_{e}}(s)$ and $H_{a_{z_{c g}}, \delta_{e}}(s)$, respectively. Note that the controlled dynamics $H_{c}(s)$ are a combination of the FBW system dynamics and the pitch response of the aircraft. Furthermore, note that the total heave at the pilot station $a_{z}$ is the summation of c.g. heave $a_{z_{c g}}$ and pitch heave $a_{z_{\theta}}$, as defined in Eq. (1).

The pitch attitude $\theta$ and the target forcing function $f_{t}$ are used to calculate the error $e$, displayed on the compensatory display. In addition, pitch and heave accelerations serve as inputs to the simulator motion system. For the purpose of this experiment, the dynamics of both the display and motion system ${ }^{23}$ can be approximated by a pure time delay, $\tau_{d s}$ and $\tau_{m s}$, respectively. In the heave motion channel a motion filter is incorporated with dynamics $H_{m f}(s)$.

The simulator display and motion systems provide the pilot with visual cues $e$, and physical motion cues $\ddot{\theta}_{s}$ and $a_{z_{s}}$, respectively. The pilot's responses to these different cues can be modeled by linear response functions. $H_{p e}(s)$ is the linear response to visual error cues. $H_{p \theta}(s)$ describes the response to rotational accelerations using the semicircular canals, while $H_{p a_{z}}(s)$ describes the response to linear accelerations using the otoliths. ${ }^{24,25}$ The pilot control output $u_{y}$ is a summation of the outputs of the linear response functions and a remnant signal $n$ that accounts for nonlinear behavior. This pilot control output results in another control input through the sidestick, effectively closing the control loop.

The control scheme of Figure 2 directly translates to the control scheme of the pitch target-following disturbance-rejection task in the aircraft. In this case, the FBW system and aircraft dynamic models are replaced by the actual FBW system and the aircraft. Note that no motion system dynamics are present in the control scheme, however, there are still display dynamics in the form of a time delay.

\section{II.C. Controlled Dynamics}

The total controlled dynamics are a combination of the FBW system and the aircraft pitch dynamics. A nonlinear dynamic model of the FBW control system was implemented in the SRS. ${ }^{26}$ The FBW system is a nonlinear system in part due to several limits on internal control signals. These limits are either physical limits of system components or limits introduced for safety reasons. As multimodal pilot models are based on linear response functions, these limits should be avoided as much as possible by proper experiment design. Extensive limiting will increase the nonlinear part of the pilot's control output, reducing the accuracy of the estimated linear response functions. ${ }^{15}$ When the FBW system is operated within its limits, the dynamics of the FBW pitch channel $H_{f b w}(s)$ can be simplified to a gain of 0.045 and a time delay of $60 \mathrm{~ms}$ as identified using experimental data in Ref. 27.

As there was no nonlinear model available of the Cessna Citation II, the aircraft dynamics in the SRS were represented by linear transfer functions. This is justified by the fact that there were only small deviations from the trim condition during an experiment run. Before the simulator part of the experiment was conducted, the aircraft pitch and c.g. heave dynamics were identified using data from a test flight. The dynamics were identified for an indicated airspeed of $160 \mathrm{kt}$ and an altitude of 17,000 ft. This was determined to be the optimal flight condition based on the properties of the FBW system, weather conditions, and availability of controlled airspace.

A frequency-domain criterion was minimized to fit the transfer function of a two degree-of-freedom shortperiod approximation to an identified frequency response. ${ }^{28}$ This frequency response was an autoregressive exogeneous (ARX) model estimate calculated using flight test data of the elevator $\delta_{e}$, pitch angle $\theta$, and c.g. acceleration $a_{z_{c g}}$. The identified transfer functions are given by:

$$
\begin{gathered}
H_{\theta, \delta_{e}}(s)=K_{\theta, \delta_{e}} \frac{T_{\theta_{2}} s+1}{s\left(\frac{s^{2}}{\omega_{s p}^{2}}+\frac{2 \zeta_{s p}}{\omega_{s p}} s+1\right)}=1.64 \frac{0.90 s+1}{s\left(\frac{s^{2}}{5.49}+0.41 s+1\right)} \\
H_{a_{z_{c g}}, \delta_{e}}(s)=K_{h, \delta_{e}} \frac{\left(T_{h_{2}} s+1\right)\left(T_{h_{3}} s+1\right)}{\frac{s^{2}}{\omega_{s p}^{2}}+\frac{2 \zeta_{s p}}{\omega_{s p}} s+1}=157.39 \frac{(0.15 s+1)(-0.16 s+1)}{\frac{s^{2}}{5.49}+0.41 s+1}
\end{gathered}
$$


The controlled pitch dynamics are a single integrator for lower frequencies up to $1 / T_{\theta_{2}}=1.12 \mathrm{rad} / \mathrm{s}$. Then, up to a peak resulting from the short-period eigenmode at $\omega_{s p}=\sqrt{5.49}=2.34 \mathrm{rad} / \mathrm{s}$, the aircraft pitch dynamics are a gain. Finally, for frequencies above the short-period eigenfrequency, the system behaves like a double integrator.

\section{II.D. Forcing Functions}

As depicted in Figure 2, disturbance and target forcing function signals $f_{d}$ and $f_{t}$ were used for inducing pilot control activity. Both the target and disturbance forcing functions were constructed as quasi-random sums of 10 sinusoids, according to:

$$
f_{d, t}(t)=\sum_{k=1}^{10} A_{d, t}(k) \sin \left[\omega_{d, t}(k) t+\phi_{d, t}(k)\right]
$$

Tracking runs were defined to last a total of $90 \mathrm{~s}$, of which the final $81.92 \mathrm{~s}$ were considered as the measurement interval $T_{m}$. Sinusoid frequencies $\omega_{d, t}(k)$ were distributed over the frequency range of interest $(0.1-20 \mathrm{rad} / \mathrm{s})$ and were defined to be integer multiples of the measurement time base frequency $\omega_{m}=2 \pi / T_{m}$ : $\omega_{d, t}(k)=n_{d, t}(k) \omega_{m}$. The integer factors $n_{d}$ and $n_{t}$ were chosen such that $f_{d}$ and $f_{t}$ had power at interleaving frequencies to allow for multimodal pilot model identification using spectral methods. ${ }^{3}$

To yield forcing function signals with reduced power at the higher frequencies, the amplitude distributions $A_{d, t}(k)$ were defined using the low-pass filter described in Ref. 4:

$$
A_{d, t}(k)=\left|\frac{\left(1+T_{A_{1}} j \omega_{d, t}(k)\right)^{2}}{\left(1+T_{A_{2}} j \omega_{d, t}(k)\right)^{2}}\right|
$$

The time constants $T_{A_{1}}$ and $T_{A_{2}}$ in Eq. (5) were set to 0.1 and $0.8 \mathrm{~s}$, respectively. The forcing function signal phases $\phi_{d, t}(k)$ were chosen randomly, though care was taken not to end up with signals with severe cresting or peaking due to phase overlap. ${ }^{29}$ The target forcing function signal was scaled to have a timedomain variance of $0.4 \mathrm{deg}^{2}$. The disturbance forcing function amplitudes as defined by Eq. (5) were pre-shaped with the inverse of the combined aircraft and FBW system dynamics (see Figure 2) and scaled to yield a low-pass disturbance of the pitch attitude $\theta$ with a time-domain variance of $0.4 \mathrm{deg}^{2}$. Note that as control inputs to the FBW system were given in Volts, the resulting disturbance signal also has that unit.

The numerical values of all multi-sine forcing function signal parameters defined in Eq. (4) are listed in Table 1. Figure 4 depicts the first 30 seconds of both forcing function signals. Note the different axes for both forcing functions.

Figure 4 shows a low-frequency target forcing function $f_{t}$ and a much more high-frequency $f_{d}$, which is a result of the pre-shaping filter. Furthermore, note that a fade-in was applied to the disturbance signal so that $f_{d}$ would gently increase to its full amplitude over the first 5 seconds of the run-in time of each tracking run. Figure 4 further shows that the target forcing function that needed to be tracked by the pilots was defined with respect to the trim pitch attitude of the aircraft. During the in-flight experiments, this $\theta_{0}$ was determined from the actual aircraft pitch at the start of each run. For the simulator experiments, $\theta_{0}$ was the fixed aircraft model trim pitch that resulted from the selected trim condition and which was also used for pitch attitude motion cueing (see Section III.A.3).

Table 1. Experiment forcing function properties.

\begin{tabular}{rrrrrrrrl}
\hline \hline \multirow{2}{*}{$k,-$} & \multicolumn{3}{c}{ Disturbance, $f_{d}$} \\
\cline { 2 - 9 } & $n_{d},-$ & $\omega_{d}, \mathrm{rad} \mathrm{s}^{-1}$ & $A_{d}, \mathrm{~V}$ & $\phi_{d}, \mathrm{rad}$ & $n_{t},-$ & $\omega_{t}, \mathrm{rad} \mathrm{s}^{-1}$ & $A_{t}, \mathrm{deg}$ & $\phi_{t}, \mathrm{rad}$ \\
\hline 1 & 5 & 0.383 & 0.026 & 1.145 & 6 & 0.460 & 0.698 & 1.288 \\
2 & 11 & 0.844 & 0.035 & 5.336 & 13 & 0.997 & 0.489 & 6.089 \\
3 & 23 & 1.764 & 0.021 & 0.802 & 27 & 2.071 & 0.220 & 5.507 \\
4 & 37 & 2.838 & 0.017 & 7.390 & 41 & 3.145 & 0.119 & 1.734 \\
5 & 51 & 3.912 & 0.021 & 8.326 & 53 & 4.065 & 0.080 & 2.019 \\
6 & 71 & 5.446 & 0.028 & 5.398 & 73 & 5.599 & 0.049 & 0.441 \\
7 & 101 & 7.747 & 0.038 & -1.349 & 103 & 7.900 & 0.031 & 5.175 \\
8 & 137 & 10.508 & 0.053 & 0.128 & 139 & 10.661 & 0.023 & 3.415 \\
9 & 171 & 13.116 & 0.071 & 0.696 & 194 & 14.880 & 0.018 & 1.066 \\
10 & 226 & 17.334 & 0.109 & 0.916 & 229 & 17.564 & 0.016 & 3.479 \\
\hline \hline
\end{tabular}




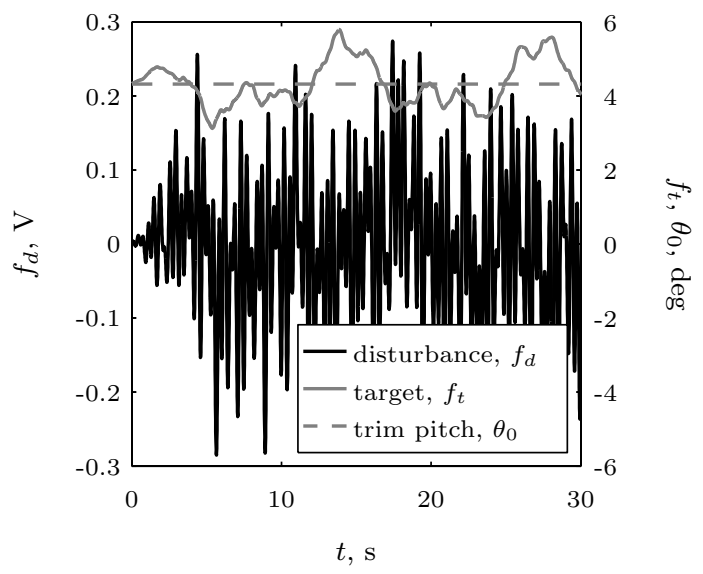

Figure 4. Forcing function time traces.

\section{II.E. Multimodal Pilot Model}

Human operators subconsciously adjust their control behavior to the controlled dynamics in such a way that the pilot-aircraft open-loop dynamics in the region of crossover can be described by integrator dynamics and a time delay. ${ }^{30}$ Figure 5 provides an appropriate model structure to model multimodal pilot control behavior in the pitch attitude control task given in Figure 2. ${ }^{24,25}$ The model consists of two inputs $e$ and $\theta$ and two channels $H_{p e}(s)$ and $H_{p \theta}(s)$ to model the pilot's visual and vestibular modalities. A remnant signal $n$, which can be characterized by a low-pass filtered white noise signal, is added to the output of the linear channels to account for nonlinearities in the pilot control output.

Note that the model only consists of two channels as opposed to the three channels in Figure 2. It has been shown in previous experiments that both the semicircular canals and the otoliths provide additional pilot lead - that is, a response to rotational velocity - in parallel to the lead generated by the visual response. ${ }^{24,31}$ Due to the similar contribution of these two channels to the overall pilot response, the total model would be overdetermined, decreasing the accuracy of the model parameters. Therefore, the contributions of the vestibular pitch and heave motion channels were combined in a single vestibular response channel that takes the form of a pure lead with a time delay. ${ }^{5}$

With the aircraft pitch dynamics provided by Eq. (2) and typical crossover frequencies between 2 and 4 $\mathrm{rad} / \mathrm{s}$ for this type of control task, the pilot needs to generate lag around $1 / T_{\theta_{2}}$ to compensate for the gain dynamics before the short-period frequency. Subsequently, a quadratic lead term is needed around $\omega_{s p}$ to compensate for the lag and achieve the required lead compensation for the double integrator dynamics above the short-period frequency. ${ }^{32}$ This results in the pilot equalization given in Figure 5.

The visual perception channel $H_{p e}(s)$ contains the visual gain $K_{v}$, visual lead time constant $T_{\text {lead }}$, visual lag time constant $T_{l a g}$, and visual time delay $\tau_{v}$. The vestibular channel $H_{p \theta}(s)$ includes the vestibular gain $K_{m}$ and a vestibular time delay $\tau_{m}$. In both channels, the control action of the pilot is limited by the neuromuscular system dynamics characterized by the neuromuscular damping ratio $\zeta_{n m}$ and natural frequency $\omega_{n m}$. These eight parameters were estimated in a time-domain maximum likelihood estimation (MLE) procedure to quantify changes in pilot control strategy in the different experimental conditions. ${ }^{22}$

In the frequency domain, pilot performance in attenuating the target and disturbance signals is determined by the crossover frequencies and phase margins of the target and disturbance open-loop responses, respectively. ${ }^{2}$ Using the control scheme in Figure 2 and the pilot response functions given in Figure 5, the disturbance and target open-loop responses are determined by:

$$
\begin{gathered}
H_{o l, d}(s)=\frac{U(s)}{\delta_{e}(s)}=\left[H_{p e}(s)+H_{p \theta}(s)\right] K_{s} H_{c}(s) \\
H_{o l, t}(s)=\frac{E(s)}{\theta(s)}=\frac{H_{p e}(s) K_{s} H_{c}(s)}{1+H_{p \theta}(s) K_{s} H_{c}(s)}
\end{gathered}
$$




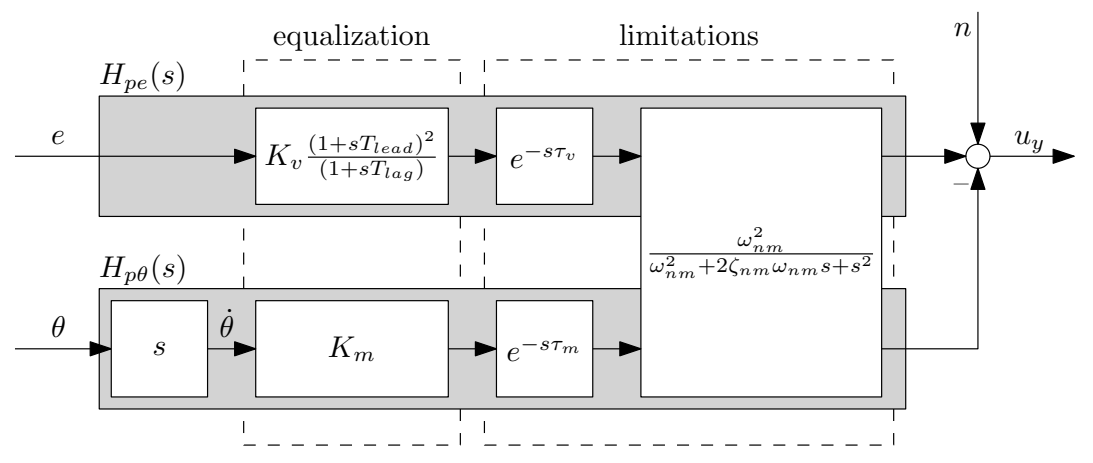

Figure 5. The multimodal pilot model structure.

The disturbance and target crossover frequencies, $\omega_{c, d}$ and $\omega_{c, t}$, are the frequencies where the magnitude of the disturbance and target open-loop responses have a magnitude of 1.0. The corresponding phase margins, $\varphi_{m, d}$ and $\varphi_{m, t}$, are the phase differences from -180 degrees at these crossover frequencies.

\section{II.F. Variables Affecting the Pilot-Vehicle System}

The experiment described in this paper attempts a comparison of pilot control behavior in two different environments: a real in-flight cockpit and a motion-base flight simulator. As pointed out by McRuer and Jex, ${ }^{18}$ human operator behavior for a certain control task is affected by a multitude of variables. These variables can be divided into four groups: environmental variables, operator-centered variables, procedural variables, and task variables. Differences in pilot control behavior resulting from differences in variables other than the change in motion fidelity would complicate the interpretation of the effect of motion cueing on pilot behavior as made in this paper and should be minimized as much as possible.

Task variables - such as, forcing functions and controlled dynamics - and procedural variables - for example, instructions and training - are relatively easy to match between the aircraft and the simulator with good experiment design. However, environmental variables - temperature, ambient lighting conditions, etc. - and operator-centered variables - such as, motivation and workload - are much more difficult to control. Another major operator-centered variable that could affect the results in the current experiment is the psychological effect of controlling an actual aircraft as opposed to controlling an aircraft model in the relatively safe environment of a flight simulator.

\section{Experiment}

The experiment setup in both the simulator and the aircraft will be discussed in the following two subsections. Next, some remaining experimental considerations, the dependent measures and hypotheses of the overall experiment, will be discussed.

\section{III.A. Simulator Experiment Setup}

III.A.1. Apparatus

The flight simulator part of the experiment was performed in the SRS at the Faculty of Aerospace Engineering (Figure 6). The SRS has a hydraulic six degree-of-freedom hexapod motion system, which was used to supply pilots with pitch and heave motion. The time delay of the SRS motion cues is $\tau_{m s} \approx 30 \mathrm{~ms}$ in all axes. ${ }^{33}$

The pilots were seated in the right pilot seat in the SRS cockpit. The compensatory display (Figure 3) was shown on the primary flight display (PFD) directly in front of them. No other visual cues, for instance from the outside visual system, were provided. The time delay of the image generation on the PFD has been measured to be in the order of $\tau_{d s} \approx 25 \mathrm{~ms}^{34}$

The pilots used a Moog FCS Ecol-8000 electrical sidestick to make control inputs. The characteristics of this control loaded sidestick can be fully adjusted. The sidestick was configured to have the same character- 


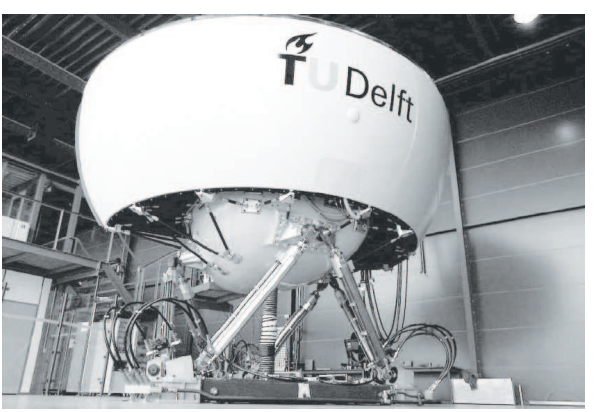

Figure 6. The SIMONA Research Simulator (SRS).

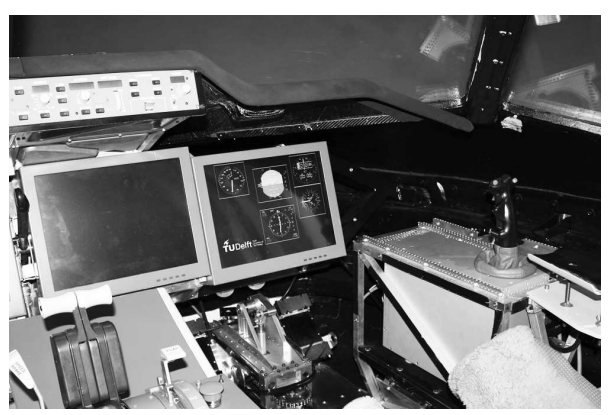

Figure 7. The SRS cockpit setup.

istics as the Citation force stick, that is, a linear force-voltage relation with a gradient of $23 \mathrm{~N} / \mathrm{V}$, limited at $\pm 2.5 \mathrm{~V}$. The stick gain $K_{s}$ (see Figure 2) was set to 0.3 .

\section{III.A.2. Independent Variables}

Four experimental conditions were evaluated in the SRS (see Table 2). In the remainder of this paper, these conditions will be referred to using the symbols listed in the first column of Table 2. A reference condition (S0) was performed without any motion cueing. In the remaining motion conditions (S1-S3), rotational pitch motion was presented 1-to-1 (no washout). In addition to the rotational pitch motion, conditions S1 and S2 presented only the pitch heave component of the total aircraft heave motion, while the total aircraft heave motion was presented in condition S3. For attenuating the total aircraft heave motion so that it could be presented using the SRS motion base, the heave washout filter also used in Ref. 4 was applied for condition S3. The same filter was also used for presenting filtered pitch heave motion in condition S1. These motion conditions represent a subset of the conditions previously evaluated for a very similar pitch control task in Refs. 4 and 5.

Table 2. Simulator experimental conditions.

\begin{tabular}{llllll}
\hline \hline \multirow{2}{*}{ Condition } & \multirow{2}{*}{ Apparatus } & \multirow{2}{*}{ Environment } & \multicolumn{3}{c}{ Motion } \\
\cline { 4 - 6 } & & & Pitch & Pitch heave & C.g. heave \\
\hline S0 & SRS & on ground & - & - & - \\
S1 & SRS & on ground & full & filtered & - \\
S2 & SRS & on ground & full & full & - \\
S3 & SRS & on ground & full & filtered & filtered \\
\hline \hline
\end{tabular}

\section{III.A.3. Simulator Motion Cueing}

By implementing additional heave and surge motion, the axis around which rotational pitch motion cues were presented was moved $0.215 \mathrm{~m}$ in lateral direction and $-0.2025 \mathrm{~m}$ in vertical direction to the same position relative to the pilot head position as it was measured to be in the Citation. Furthermore, using a prepositioning filter, the SRS was moved to the trim pitch attitude $\theta_{0}$ before the start of each measurement run. ${ }^{35}$ Note that this prepositioning was also done for the no-motion condition S0. The prepositioning filter was defined as:

$$
H_{p p}(s)=\frac{s^{2}}{s^{2}+2 \zeta_{p p} \omega_{n_{p p}} s+\omega_{n_{p p}}^{2}} \cdot \frac{s}{s+\omega_{b_{p p}}}
$$

The parameter values of Eq. (8) can be found in Table 3, in addition to the trim pitch attitude $\theta_{0}$ to which the simulator was prepositioned. Figure 8 depicts the first part of the time trace of the simulator pitch motion response for a typical experiment run. It clearly shows the prepositioning filter response for $t<0$ and the simulator pitch motion resulting from the pitch control task for $t>0$. Due to the prepositioning and the moderate magnitude of the pitch excursions attained during the control task, the simulator pitch attitude was always positive during the whole experiment. 
Table 3. Motion cueing parameters.

\begin{tabular}{llll}
\hline \hline Filter & Parameter & Value & Unit \\
\hline \multirow{4}{*}{$H_{p p}$} & $\omega_{n p p}$ & 1.0 & $\mathrm{rad} \mathrm{s}^{-1}$ \\
& $\zeta_{p p}$ & 1.0 & - \\
& $\omega_{b_{p p}}$ & 2.0 & $\mathrm{rad} \mathrm{s}^{-1}$ \\
& $\theta_{0}$ & 4.34 & $\mathrm{deg}$ \\
\hline \multirow{4}{*}{$H_{m f}$} & $K_{m f}$ & 0.6 & - \\
& $\omega_{n}$ & 1.25 & $\mathrm{rad} \mathrm{s}^{-1}$ \\
& $\zeta_{m f}$ & 0.7 & - \\
& $\omega_{b m f}$ & 0.3 & $\mathrm{rad} \mathrm{s}^{-1}$ \\
\hline \hline
\end{tabular}

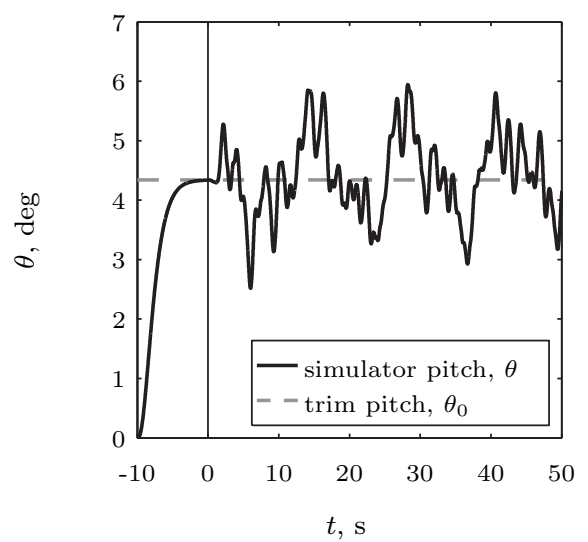

Figure 8. Time trace of simulator pitch attitude.

For two of the SRS conditions (S1 and S3) a heave motion filter was used for presenting heave motion cues (Table 2). The filter that was used for these conditions was the same third-order high-pass filter also utilized in Ref. 4:

$$
H_{m f}(s)=K_{m f} \frac{s^{2}}{s^{2}+2 \zeta_{m f} \omega_{n_{m f}} s+\omega_{n_{m f}}^{2}} \cdot \frac{s}{s+\omega_{b_{m f}}}
$$

The values for the filter corner frequencies, $\omega_{n_{m f}}$ and $\omega_{b_{m f}}$, and damping factor $\zeta_{m f}$ that were used for the experiment can be found in Table 3 .

\section{III.A.4. Experimental Procedures}

All pilots performed the simulator part of the experiment before the in-flight part in the Citation laboratory aircraft. Pilots performed a number of training runs, typically 4-6 repetitions of each experimental condition, until their proficiency in performing the tracking task had stabilized. Then five repetitions of each experimental condition were collected as the measurement data. The motion conditions listed in Table 2 (S0-S3) were presented in random order (Latin square) throughout both the training and measurement phases of the experiment. Breaks were taken regularly to avoid fatigue.

After the simulator was prepositioned to the aircraft trim pitch attitude, the experimenter counted down from three and started the run. Directly after a run ended the simulator was tilted back to zero pitch attitude, after which participants were asked to give a subjective judgment of motion fidelity for the run they just completed (see Section III.D). After this subjective evaluation was completed pilots were informed of their tracking score, defined as the root mean square (RMS) of the recorded error signal $e$.

\section{III.B. Aircraft Experiment Setup}

\section{III.B.1. Apparatus}

The Cessna Citation II laboratory aircraft depicted in Figure 9 is jointly owned by the Faculty of Aerospace Engineering of TU Delft and the Netherlands' National Aerospace Laboratory (NLR). It is a twin-jet business aircraft powered by Pratt \& Whitney JT15D-4 turbofan engines. The maximum operating altitude of the aircraft is $43,000 \mathrm{ft}$ and the maximum cruising speed is $385 \mathrm{kt}$. In addition to a custom Flight Test Instrumentation System (FTIS) that is available in the aircraft, ${ }^{17}$ additional sensors can be installed on a nose boom, roof rack, or external pod underneath the aircraft.

The experiment setup in the laboratory aircraft was very similar to that used for the experiment of Ref. 27. A FBW control system has been especially developed and installed into the aircraft for the purpose of this experiment and has been extensively described in Ref. 17. The aircraft was equipped with a nose boom with alpha and beta vanes, which allowed for accurate monitoring of the angle of attack and sideslip, respectively. 


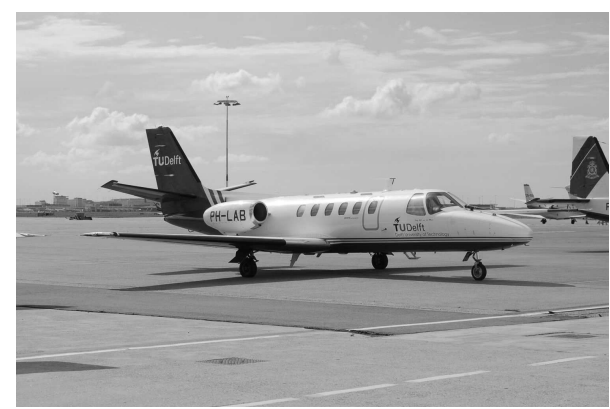

Figure 9. The Cessna Citation II laboratory aircraft.

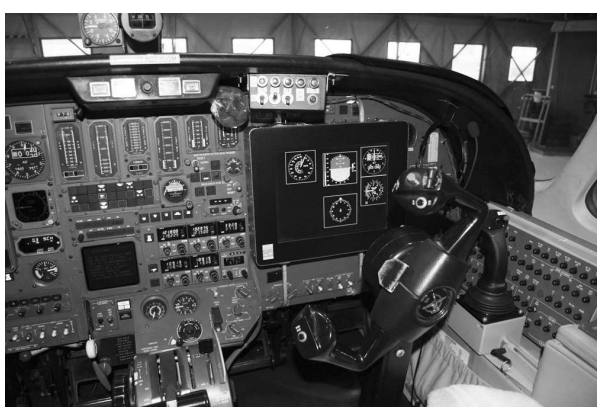

Figure 10. The Cessna Citation II cockpit setup.

As can be verified from Figure 10, the setup in the Citation cockpit was very similar to that in the SRS cockpit. The experiment was performed from the right pilot seat. A programmable LCD display was installed in front of the original instrument panel on which the compensatory display shown in Figure 3 was presented. Using a custom visual delay measurement system, ${ }^{34}$ the latency of the display (including the projection) was measured to be $\tau_{d s} \approx 25 \mathrm{~ms}$. This is approximately the same as the delay found for the display in the simulator.

Figure 10 further shows the sidestick manipulator that was installed at the right pilot seat in the Citation cockpit. Due to the limited space available in the cockpit, a BG Systems JFf force joystick was used to make control inputs during the in-flight tracking tasks. As opposed to a deflection stick, a force stick has very high stiffness and no manipulator deflections are required for making a control input. Rather, the control input made by the pilot is proportional to the force applied to the manipulator. The output voltage of the stick was limited between $\pm 2.5 \mathrm{~V}$. The relation between applied force and output voltage was determined from static measurements, where known weights were used to induce known stick forces. The resulting output voltage was then measured. The force-voltage characteristic of the Citation force stick was found to be approximately linear with a gradient of $23 \mathrm{~N} / \mathrm{V}$ over the full range of the output voltage.

As explained in detail in Refs. 17 and 27, the side stick commands and the disturbance forcing function signal were fed to a custom experiment computer, which in turn used the actuators of the aircraft's automatic flight control system to control the control surfaces. Note that due to the fact that the automatic control system makes use of the mechanical control architecture, both side stick inputs and the disturbance forcing function signal resulted in a moving control column (see Figure 10).

\section{III.B.2. Independent Variables}

The three different conditions evaluated in the in-flight part of the experiment are listed in Table 4. These conditions will be referred to using the symbols in the first column of Table 4. To determine the effect of limited motion cues on pilot control behavior, the conditions performed in the SRS were compared with the in-flight condition CF. For this baseline condition the full unrestricted aircraft pitch and heave motion are present.

Table 4. In-flight experimental conditions.

\begin{tabular}{llllll}
\hline \hline \multirow{2}{*}{ Condition } & \multirow{2}{*}{ Apparatus } & \multirow{2}{*}{ Environment } & \multicolumn{3}{c}{ Motion } \\
\cline { 4 - 6 } & & & Pitch & Pitch heave & C.g. heave \\
\hline $\mathrm{C} 0$ & Citation & on ground & - & - & - \\
$\mathrm{COF}$ & Citation & in flight & - & - & - \\
$\mathrm{CF}$ & Citation & in flight & full & full & full \\
\hline \hline
\end{tabular}

Table 4 lists two additional conditions that were performed in the Citation laboratory aircraft: $\mathrm{C} 0$ and $\mathrm{C0F}$. For these two conditions the pitch control task was performed using the experimental setup utilized for the in-flight measurements of $\mathrm{CF}$, but pilots were controlling the same model of the Citation and FBW system dynamics as used in the SRS conditions rather than the aircraft itself. Condition C0 was performed on the ground and therefore yielded a condition that should be equivalent to the simulator condition without 
any motion, S0. Condition $\mathrm{C} 0 \mathrm{~F}$ was the same as $\mathrm{C} 0$, but the measurements for this condition were taken during flight, at the same altitude and velocity as selected for CF.

Even though extreme care was taken to ensure the important elements of the experimental setups in the Citation and SRS were as equal as possible, these additional measurements were performed to quantify possible discrepancies in control behavior due to remaining differences in the experimental setup and the environment in which pilot control was measured.

\section{III.B.3. Experimental Procedures}

As in the simulator part of the experiment, participants performed a number of training runs until their proficiency in performing the tracking task had stabilized. Then five more repetitions of each experimental condition were collected as the measurement data. The three conditions of the in-flight part of the experiment (see Table 4) were always performed in the same order.

Before take-off, the measurements for the $\mathrm{C} 0$ condition were taken. The main reason for taking these measurements first was that it allowed for initial refamiliarization with the control task in a more controlled environment than available during flight. This refamiliarization was necessary as the aircraft part of the experiment was conducted some time after the simulator part of the experiment. In addition, it was thought to reduce the number of training runs required for the in-flight measurements of condition CF. During flight, both pilots performed the in-flight aircraft control task (condition $\mathrm{CF}$ ) first, after which condition $\mathrm{COF}$ was evaluated.

During the in-flight measurements, two pilots were always required for each flight. The pilot in the left seat functioned as the safety pilot and was responsible for monitoring the aircraft during the experiment and ensured the aircraft was in the desired trim state $(V=160 \mathrm{kt}, h=17,000 \mathrm{ft})$ before each run. The other pilot, referred to as the experiment pilot, performed the experiment.

To avoid the moving control column and pilots' view of the outside world affecting their control strategy during the pitch tracking task, the experiment pilot was required to wear a hood (see Figure 11) that limited the field of view to the LCD display during the measurements for conditions $\mathrm{CF}$ and $\mathrm{C} 0 \mathrm{~F}$. For condition C0, participants did not wear the hood.

For conditions $\mathrm{C} 0$ and $\mathrm{C} 0 \mathrm{~F}$ the experimenter in the main cabin of the aircraft initiated the start of a run after counting down from three, as was also done during the simulator part of the experiment. For the CF condition, where the actual aircraft was controlled, the run was initiated by the experiment pilot using the pilot interface shown above the LCD screen in Figure 10. For more details on this pilot interface, refer to Refs. 17 and 27.

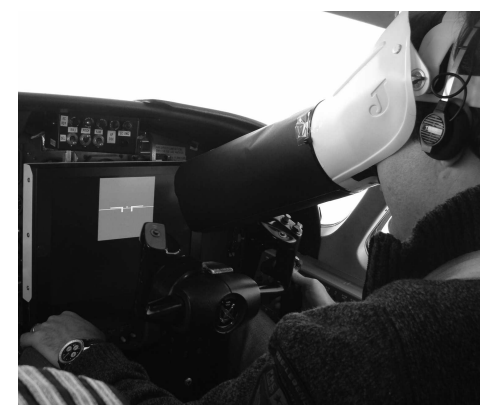

Figure 11. Experiment pilot wearing the hood during the in-flight experiment.

After completion of a run for condition CF, the FBW system would disengage itself, after which the safety pilot would take control of the aircraft and bring it back in the desired trim state for the next run of the experiment. The experimenter then notified the experiment pilot of his performance for the last run, defined as the RMS of the error signal $e$.

\section{III.C. Pilots}

Seven pilots performed the pitch attitude tracking task under the four simulator conditions listed in Table 2 and the three in-flight conditions listed in Table 4. All participants were active Cessna Citation II pilots and all except one had experience with similar control tasks from previous simulator and in-flight experiments. The participants' flight experience ranged from 1,500-14,000 hrs on a multitude of different aircraft. Their ages ranged from 34 to 72 years.

\section{III.D. Dependent Measures}

Several dependent measures were considered for this experiment to evaluate the effects of the variation in motion fidelity on pilot tracking performance and multimodal control behavior. First of all, during the SRS part of the experiment, participants were asked to give a subjective indication of the motion fidelity. Participants rated the motion fidelity by drawing a vertical line through a horizontal bar of a motion fidelity 
rating scale for each tracking run. Zero percent (the left side of the bar) indicated that the experienced simulator motion was not like the real aircraft motion at all, while $100 \%$ (the right side of the bar) indicated that the motion was perceived to be exactly the same as experienced in the aircraft. These subjective ratings merely served as a reference for the objective measures of the experiment.

In addition, a large number of objective measures of pilot tracking performance, control activity, and control behavior were considered. Tracking performance and control activity were evaluated from the timedomain variances of the recorded time traces of the pitch tracking error $e$ and pilot control signal $u_{y}$, respectively. Using a spectral method described in Ref. 2, the contributions of the target and disturbance forcing functions and pilot remnant to these signal variances were evaluated.

The most important dependent measures that were considered, however, are the parameters of the multimodal pilot model introduced in Section II.E. These pilot model parameters were identified from measurements of $e, u_{y}$, and $\dot{\theta}$ (the latter only for conditions with motion cues) using the time-domain MLE procedure described in Ref. 22. Before being used for model identification, the high-frequency noise present in these signals - that is, the noise above $30 \mathrm{rad} / \mathrm{s}$, well above the highest frequency sinusoids in $f_{t}$ and $f_{d}$ (Table 1) - was filtered out. The five repetitions of these signals were then averaged to yield one identification data set for each condition and participant. Finally, using the calculated open-loop frequency responses, pilot-vehicle system crossover frequencies and phase margins were determined.

\section{III.E. Hypotheses}

To assess possible differences in control strategy that resulted from the difference in experimental setup and environmental factors, the $\mathrm{C} 0$ and $\mathrm{C} 0 \mathrm{~F}$ no-motion conditions from the aircraft part of the experiment where compared directly to the equivalent S0 no-motion condition from the SRS. Extreme care was taken to ensure that the conditions under which the in-flight and simulator experiments were performed were as similar as possible. Hence, differences in control strategy observed between the S0, C0, and C0F conditions were expected to be minor compared to the effect of the variation in motion cueing.

The variation in simulator motion cueing evaluated for the comparison with in-flight measurements of pilot control behavior consists of cueing conditions also evaluated in previous simulator experiments. ${ }^{4,5}$ Based on the findings of these two experiments and many additional experiments performed in the past ${ }^{36}$ it is expected that pilot tracking performance and control activity will increase for increasing pitch heave motion fidelity. However, a decrease in performance is expected when c.g. heave motion is present. Consistent with the increase in performance, an increase in disturbance crossover frequency and decrease in disturbance phase margin is expected.

A change in pilot control strategy for increasing motion fidelity will be observed by an increase in visual and vestibular gains, a decrease in visual lead and lag, and an increase in visual and vestibular time delays, as observed in the previous experiments. However, as found in Ref. 4, an increase in visual lead is expected when c.g. heave is present. Due to this effect of c.g. heave, it is hypothesized that the SRS condition S3 will yield pilot control behavior that is closest to that observed in flight for condition CF.

Finally, the experiment described here allows for a direct comparison of subjective and objective measurements of flight simulator motion fidelity for the conditions evaluated in the SRS. It is expected that both these sets of dependent measures will show similar general trends. The objective measurements of pilot behavior, however, are expected to allow for better evaluation of the differences in fidelity between the motion conditions S1-S3.

\section{Results}

This section presents the combined results of the seven pilots who participated in the experiment. In figures displaying data from all experimental conditions, the baseline condition $\mathrm{CF}$ is marked by a gray horizontal line for reference. The error bar plots are corrected for between-subject variability by normalizing the subject means. ${ }^{37}$ All the calculated dependent measures were analyzed using a repeated-measures analysis of variance (ANOVA) to reveal any significant effects.

First, an ANOVA was performed on conditions $\mathrm{C} 0, \mathrm{C} 0 \mathrm{~F}$, and $\mathrm{S} 0$ - that is, the three conditions without physical motion - to determine the effects induced by the experiment environment or apparatus (see Tables 2 and 4). Second, an ANOVA was performed on conditions S0, S1, S2, S3, and CF, to reveal the effects of increasing motion fidelity. In every ANOVA, Mauchly's test of sphericity was performed to test if the 
(a) pitch magnitude

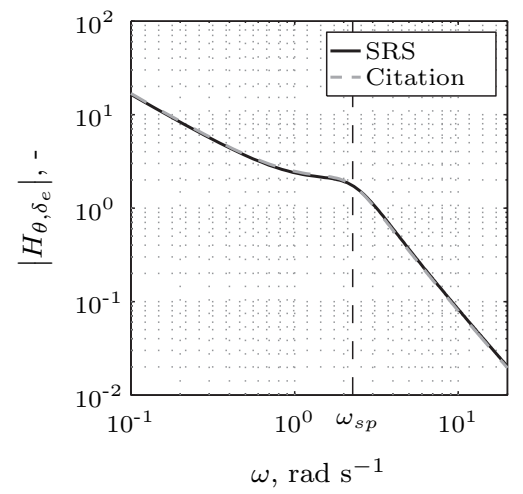

(c) pitch phase

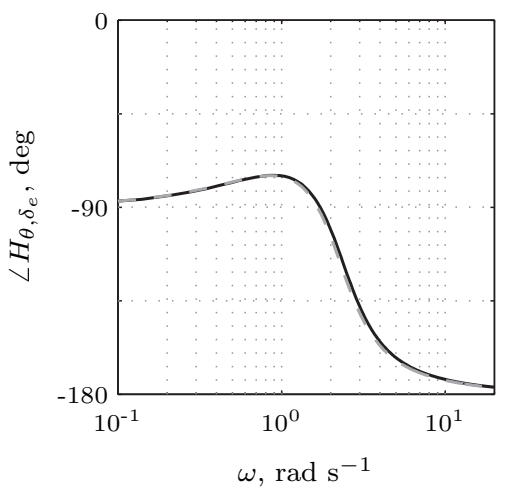

(b) c.g. heave magnitude

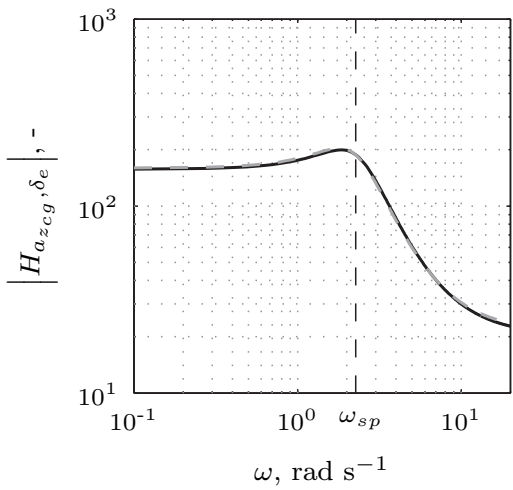

(d) c.g. heave phase

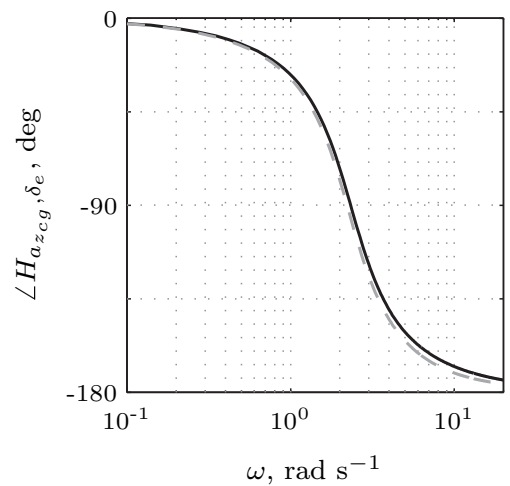

Figure 12. Mean frequency responses of the aircraft dynamics $(V=160 \mathrm{kt}$ and $h=17,000 \mathrm{ft})$.

assumption of sphericity was met $(p>0.05) .{ }^{37}$ If the assumption was not met, the degrees of freedom were corrected using Greenhouse-Geisser estimates of sphericity.

\section{IV.A. Aircraft Dynamics}

To validate that pilots controlled the same aircraft dynamics in both the simulator and the aircraft parts of the experiment, the controlled pitch dynamics were identified using data from both apparatus. An MLE procedure was used to estimate the parameters of a two degree-of-freedom short-period approximation of the aircraft dynamics. ${ }^{28}$ The mean frequency responses of the dynamic model implemented in the SRS are calculated using the data of conditions S0-S3 and all pilots, the Citation mean frequency responses are calculated using the data of condition $\mathrm{CF}$ and all pilots. The frequency responses of the aircraft pitch and c.g. heave dynamics, $H_{\theta, \delta_{e}}(j \omega)$ and $H_{a_{z_{c g}}, \delta_{e}}(j \omega)$, are depicted in Figure 12.

In the Citation part of the experiment, there were slight deviations from the desired trim condition for every experiment run. However, Figure 12 shows that the mean responses for the pitch and c.g. heave dynamics in both the SRS and the Citation are approximately equal, which means that these slight deviations likely have only a very small effect on the controlled aircraft dynamics. Given a certain input, the variance accounted for (VAF) is a measure of how well the output of the identified model describes the measured time-domain data. ${ }^{15}$ For the short-period approximations identified here, the VAF was generally above $99 \%$ for the pitch dynamics and above $96 \%$ for the c.g. heave dynamics, indicating that the accuracy of the identified models is very high.

\section{IV.B. Subjective Evaluations}

Figure 13 provides an error bar plot of the simulator motion fidelity rating results. The figure also depicts the mean data from individual pilots. The results indicate that pilots rated the condition without simulator motion ( $\mathrm{S} 0$ ) the lowest. However, one pilot consistently rated this condition with a significantly higher value compared to the other pilots. All the motion conditions (S1-S3) were rated approximately equal, around $65 \%$. 


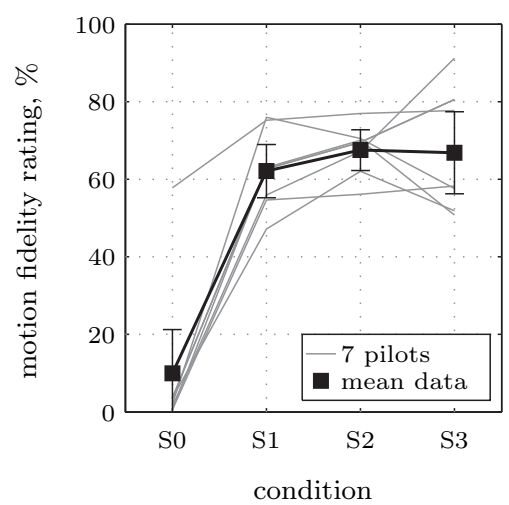

Figure 13. Subjective motion fidelity ratings.

(a) error signal

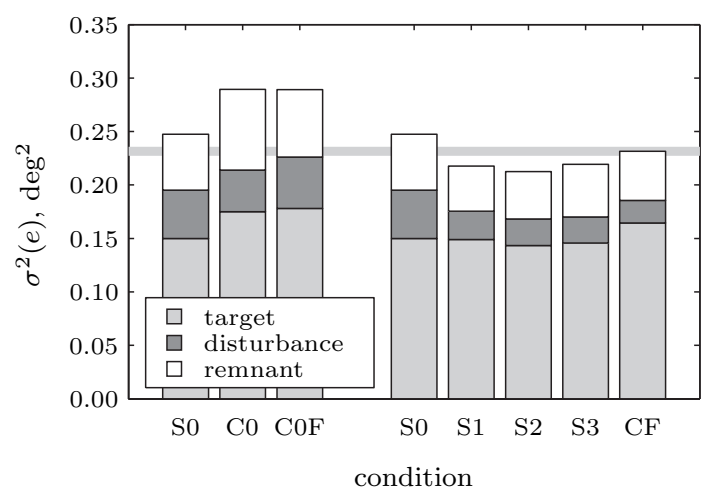

(b) control signal

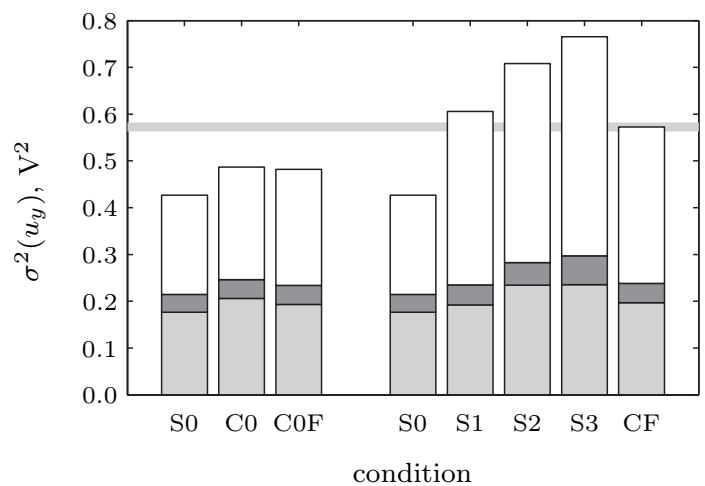

Figure 14. Variance decomposition of pilot performance and control activity.

However, the simulator condition with filtered c.g. heave (S3) shows a slightly larger error bar, indicating that pilots rated this motion condition less consistently, as can also be observed from the individual pilot data.

\section{IV.C. Pilot Performance and Control Activity}

Pilot performance and control activity were evaluated using the variance of the error and pilot control signals, respectively. The disturbance forcing function, target forcing function, and remnant portions of the variance were determined using the power spectral densities of the signals at the input frequencies of the forcing functions. ${ }^{2}$

The calculated variances for the error signal are depicted in Figure 14(a) and the corresponding ANOVA results are given in Table 5. Note that both the disturbance and target forcing functions were scaled to induce an error variance of $0.4 \mathrm{deg}^{2}$ (see Section II.D). Figure 14(a) indicates that pilots could attenuate a significantly higher percentage of the disturbance forcing function as compared to the target forcing function, as the disturbance portions of the variance bars are smaller than the target portions. This result was also found in previous experiments. ${ }^{16,27}$

The ANOVA of the total variance of the error in the no-motion conditions (Table 5) indicates that overall performance was significantly better in the simulator no-motion condition (S0) as compared to the aircraft no-motion conditions ( $\mathrm{C} 0$ and $\mathrm{COF}$ ). The ANOVA further indicates that the disturbance component of the error variance is not significantly affected by the experiment environment. However, in the simulator nomotion condition, the target component of the variance was significantly lower than in the aircraft no-motion conditions. This implies that the increase in overall pilot performance in the simulator was caused by a better attenuation of the target signal.

The level of motion fidelity (conditions S0-S3, and CF) was also found to have a significant effect on overall performance. Figure 14(a) indicates a slight improvement in performance (decreasing error variance) when 
Table 5. ANOVA results of pilot performance, where $* *$ is highly significant $(p<0.01), *$ is significant $(0.01 \leq$ $p<0.05)$, and - is not significant $(p \geq 0.05)$.

\begin{tabular}{|c|c|c|c|c|c|c|c|c|c|}
\hline \multirow{2}{*}{$\begin{array}{c}\text { Independent } \\
\text { variables }\end{array}$} & \multicolumn{9}{|c|}{ Dependent measures } \\
\hline & \multicolumn{3}{|c|}{$\sigma^{2}(e)$} & \multicolumn{3}{|c|}{$\sigma^{2}\left(e_{d}\right)$} & \multicolumn{3}{|c|}{$\sigma^{2}\left(e_{t}\right)$} \\
\hline Factor & $\mathrm{df}$ & $\mathrm{F}$ & Sig. & $\mathrm{df}$ & $\mathrm{F}$ & Sig. & $\mathrm{df}$ & $\mathrm{F}$ & Sig. \\
\hline Environment $(\mathrm{S} 0, \mathrm{C} 0, \mathrm{C} 0 \mathrm{~F})$ & 2,12 & 7.355 & $* *$ & 2,12 & 1.000 & - & 2,12 & 5.716 & * \\
\hline Motion $(\mathrm{S} 0, \mathrm{~S} 1, \mathrm{~S} 2, \mathrm{~S} 3, \mathrm{CF})$ & 4,24 & 5.694 & $* *$ & 4,24 & 13.651 & $* *$ & $1.9,11.3$ & 4.746 & * \\
\hline
\end{tabular}

Table 6. ANOVA results of pilot control activity, where $* *$ is highly significant $(p<0.01), *$ is significant $(0.01 \leq p<0.05)$, and - is not significant $(p \geq 0.05)$.

\begin{tabular}{lcccccccccc}
\hline \hline \multirow{2}{*}{$\begin{array}{c}\text { Independent } \\
\text { variables }\end{array}$} & \multicolumn{9}{c}{ Dependent measures } \\
\cline { 2 - 11 } & $\mathrm{df}$ & $\mathrm{F}$ & Sig. & $\mathrm{df}$ & $\mathrm{F}$ & Sig. & $\mathrm{df}$ & $\mathrm{F}$ & Sig. \\
\hline Factor & 2,12 & 0.080 & - & 2,12 & 0.048 & - & 2,12 & 0.115 & - \\
\hline Environment $(\mathrm{S} 0, \mathrm{C} 0, \mathrm{C} 0 \mathrm{~F})$ & $\left.u_{y}\right)$ & \multicolumn{1}{c}{$\left.u_{y, t}\right)$} \\
Motion $(\mathrm{S} 0, \mathrm{~S} 1, \mathrm{~S} 2, \mathrm{~S} 3, \mathrm{CF})$ & $1.3,8.0$ & 1.869 & - & 4,24 & 4.126 & $*$ & $1.2,7.3$ & 1.313 & - \\
\hline \hline
\end{tabular}

the fidelity of pitch heave is increased and a decrease in performance (increasing variance) when filtered c.g. heave is present. This was also observed in the experiment described in Ref. 4. The disturbance component significantly decreases when the level of motion fidelity is increased from S0 to CF, indicating that pilots were better able to attenuate the disturbance signal. The main decrease in disturbance variance is present between the no-motion and the motion conditions. In the aircraft condition $\mathrm{CF}$, the target component is significantly higher than in the simulator conditions S0-S3.

Figure 14(b) depicts the variances of the control signal. The corresponding ANOVA results are given in Table 6. No significant effects were found, except for the disturbance component of the control signal in the motion fidelity conditions. In simulator condition S3 the variance of the disturbance component was significantly higher compared to the other conditions. Although not significant, an increase in total variance of the control signal can be observed for the simulator conditions for increasing motion fidelity, followed by a decrease of control activity in the in-flight condition CF.

\section{IV.D. Pilot Control Behavior}

The eight parameters of the multimodal pilot model depicted in Figure 5 were estimated using an MLE procedure on averaged time-domain data from the SRS and Citation conditions. ${ }^{22}$ For the conditions without motion, the pilot model only contained the visual channel, and the number of parameters to be identified was reduced from eight to six. As required by the MLE procedure, the pilot model was converted to a state-space representation in which the time delays were implemented using fifth order Padé approximations.

Fifty iterations were performed with a genetic MLE algorithm, after which the solutions were further optimized using a gradient-based Gauss-Newton optimization. The solution with the lowest likelihood that occurred multiple times - indicating, a consistent global minimum in the parameter search space - was chosen as the final parameter set. For all conditions of every subject a stable global minimum was found.

Figure 15 depicts the frequency responses of the pilot model visual and vestibular channels for condition CF performed by pilot 4 . The Fourier coefficients (FC) of the pilot response functions can be calculated from the measured time-domain data independent of the selected model structure and are also given in the figure. ${ }^{15}$ The estimated pilot model responses closely follow the calculated FC frequency responses, indicating a high accuracy of the model in the frequency domain. The data presented in Figure 15 are representative for all conditions and all pilots.

The VAF of the pilot model output is calculated as a measure of accuracy in the time domain. Figure 16 depicts the means and 95\% confidence intervals of the pilot model VAFs. The total VAFs for all conditions are in the order of $85 \%$ and no significant trends were found for changes in experiment environment and apparatus, and motion fidelity $[\mathrm{F}(2,12)=1.874, p>0.05$ and $\mathrm{F}(1.7,10.1)=0.681, p>0.05]$. This indicates that the multimodal pilot model can accurately describe the pilot control signal data measured in both the SRS and the aircraft equally well. Also, in both the SRS and the aircraft, $85 \%$ of the pilot control output can be described by linear response functions, revealing that pilots controlled with the same level of linearity in both apparatus. 
(a) visual magnitude

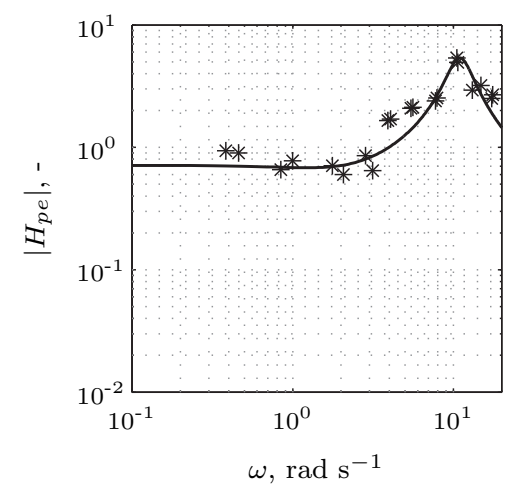

(c) visual phase

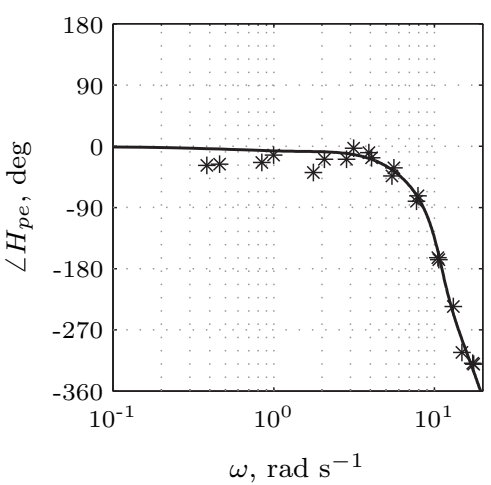

(b) vestibular magnitude

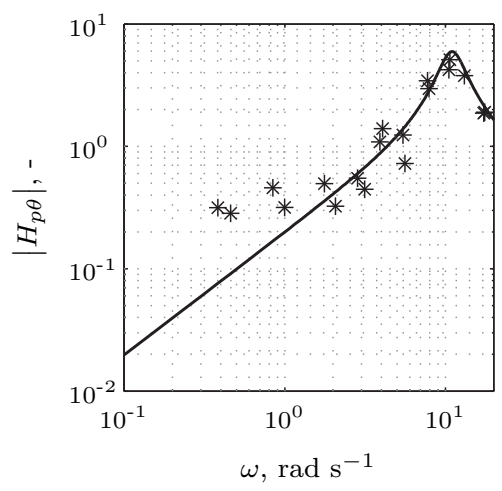

(d) vestibular phase

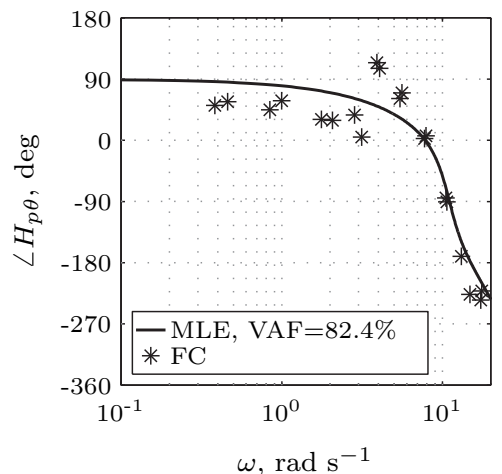

Figure 15. Frequency responses of pilot visual and vestibular modalities (CF, pilot 4).

Table 7. ANOVA results of pilot model equalization parameters, where $* *$ is highly significant $(p<0.01), *$ is significant $(0.01 \leq p<0.05)$, and - is not significant $(p \geq 0.05)$.

\begin{tabular}{|c|c|c|c|c|c|c|c|c|c|c|c|c|}
\hline \multirow{2}{*}{$\begin{array}{c}\text { Independent } \\
\text { variables }\end{array}$} & \multicolumn{12}{|c|}{ Dependent measures } \\
\hline & \multicolumn{3}{|c|}{$K_{v}$} & \multicolumn{3}{|c|}{$K_{m}$} & \multicolumn{3}{|c|}{$T_{\text {lead }}$} & \multicolumn{3}{|c|}{$T_{l a g}$} \\
\hline Factor & df & $\mathrm{F}$ & Sig. & $\mathrm{df}$ & $\mathrm{F}$ & Sig. & df & $\mathrm{F}$ & Sig. & $\mathrm{df}$ & $\mathrm{F}$ & Sig. \\
\hline Environment (S0,C0,COF) & 2,12 & 0.680 & - & & & & 2,12 & 4.675 & $*$ & 2,12 & 0.661 & - \\
\hline Motion (S0,S1,S2,S3,CF) & 4,24 & 4.430 & $* *$ & $1.3,7.9$ & 2.764 & - & 4,24 & 60.957 & $* *$ & 4,24 & 26.232 & $* *$ \\
\hline
\end{tabular}

In addition to the total VAFs, Figure 16 depicts the VAF contributions of the visual and vestibular modalities for the conditions with motion (S1-CF). Note that in the conditions without motion the contribution of the visual channel is equal to the total VAF and there is no contribution of the vestibular channel. In the conditions with motion, around $65 \%$ of the pilot output is acounted for by the visual channel and $20 \%$ by the vestibular channel. This indicates that pilots are mostly relying on their visual modality. Motion fidelity did not introduce a significant effect in the visual $\operatorname{VAF}[\mathrm{F}(1.6,9.5)=4.042, p>0.05]$, however, a significant effect was found in the vestibular $\operatorname{VAF}[\mathrm{F}(3,18)=5.348, p<0.01]$. When the fidelity of pitch heave is improved from S1 to S2, the vestibuar VAF significantly increases, indicating that pilots rely more on motion cues. However, for the simulator condition with filtered c.g. heave (S3) the vestibular VAF decreases again, to the same level as in the aircraft condition (CF).

Means and 95\% confidence intervals of the estimated multimodal pilot model parameters are depicted in Figure 17. The ANOVA results for the equalization parameters of the pilot model are summarized in Table 7. Figure $17(\mathrm{a})$ depicts the data for the visual gain $K_{v}$. This parameter was not significantly affected by the experiment environment. However, there was a significant effect of motion fidelity. An increase in visual gain with increasing simulator motion fidelity can be observed, followed by a decrease for the in-flight motion condition. Figure $17(\mathrm{~b})$ depicts the data for the vestibular gain of the pilot model. Note that this parameter is only estimated for the conditions where physical motion was present. The ANOVA on the motion conditions indicates that the vestibular gain is not significantly affected by motion fidelity. 


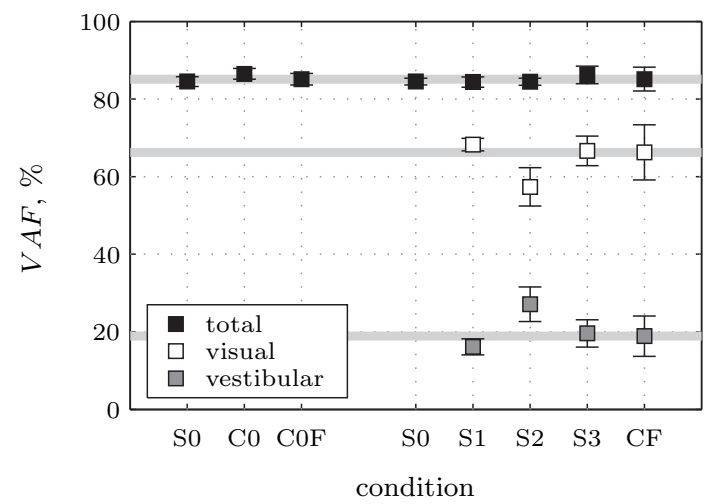

Figure 16. Pilot model VAFs.

Table 8. ANOVA results of pilot model limitation parameters, where $* *$ is highly significant $(p<0.01), *$ is significant $(0.01 \leq p<0.05)$, and - is not significant $(p \geq 0.05)$.

\begin{tabular}{|c|c|c|c|c|c|c|c|c|c|c|c|c|}
\hline \multirow{2}{*}{$\begin{array}{c}\text { Independent } \\
\text { variables }\end{array}$} & \multicolumn{12}{|c|}{ Dependent measures } \\
\hline & \multicolumn{3}{|c|}{$\tau_{v}$} & \multicolumn{3}{|c|}{$\tau_{m}$} & \multicolumn{3}{|c|}{$\zeta_{n m}$} & \multicolumn{3}{|c|}{$\omega_{n m}$} \\
\hline Factor & $\mathrm{df}$ & $\mathrm{F}$ & Sig. & $\mathrm{df}$ & $\mathrm{F}$ & Sig. & $\mathrm{df}$ & $\mathrm{F}$ & Sig. & $\mathrm{df}$ & $\mathrm{F}$ & Sig. \\
\hline Environment (S0,C0,C0F) & 2,12 & 4.317 & $*$ & & & & 2,12 & 5.601 & $*$ & 2,12 & 17.286 & $* *$ \\
\hline Motion (S0,S1,S2,S3,CF) & 4,24 & 22.693 & $* *$ & $1.3,8.1$ & 6.295 & $*$ & 4,24 & 1.033 & - & 4,24 & 2.985 & $*$ \\
\hline
\end{tabular}

Data for the visual lead and lag time constants, $T_{\text {lead }}$ and $T_{\text {lag }}$, are depicted in Figures $17(\mathrm{c})$ and $17(\mathrm{~d})$, respectively. For the control of aircraft pitch dynamics, these two equalization parameters are strongly coupled - that is, show the same trend - as can be observed from the figure. ${ }^{4,5}$ The visual lead time constant is significantly affected by the experiment environment. A slightly lower lead time constant can be observed for the in-flight no-motion condition $\mathrm{COF}$. However, the visual lag time constant is not significantly affected by experiment environment. Both $T_{l e a d}$ and $T_{l a g}$ show a highly significant decrease with the introduction of motion, indicating a reduction in the amount of visual lead equalization. This effect is mainly present between the no motion condition S0 and the remaining motion conditions S1-CF. A slight increase in both the lead and lag time constants can be observed when c.g. heave is present ( $\mathrm{S} 3$ and $\mathrm{CF}$ ). This increase was also observed in the experiment described in Ref. 4. Note that for the conditions with motion (S1-CF), the visual lead and lag time constants approximate the characteristic time constants of the controlled dynamics, $1 / \omega_{s p}$ and $T_{\theta_{2}}$, respectively. ${ }^{32}$ This shows striking evidence that pilots adjust to the controlled aircraft dynamics much more accurately when simulator motion is present.

The ANOVA results for the limitation parameters of the pilot model are given in Table 8 . The visual time delay is depicted in Figure 17(e). This parameter is significantly affected by the experiment environment. In the simulator, the visual time delay is significantly lower. In addition, motion fidelity introduces a highly significant effect. This effect reflects an increase in visual time delay for increasing levels of motion fidelity, as was also observed in a previous experiment. ${ }^{5}$ The biggest increase is present between the simulator conditions and the aircraft condition CF. Figure 17(f) depicts the results for the vestibular time delay. The vestibular delay increases for increasing motion fidelity, a significant effect. There is mainly an increase between the conditions without (S1-S2) and with c.g. heave motion (S3-CF).

The neuromuscular damping $\zeta_{n m}$ and frequency $\omega_{n m}$ - characterizing the neuromuscular dynamics - are depicted in Figures $17(\mathrm{~g})$ and $17(\mathrm{~h})$, respectively. Both the neuromuscular damping and frequency were affected by the experiment environment. For the no-motion conditions in the aircraft, $\zeta_{n m}$ and $\omega_{n m}$ are lower. In addition, the neuromuscular frequency was significantly affected in the motion fidelity conditions. It first increases for higher pitch heave motion fidelity levels, but then decreases when c.g. heave motion is present.

\section{IV.E. Crossover Frequencies and Phase Margins}

The crossover frequencies and phase margins for the disturbance and target open-loop responses - characterizing pilot-vehicle performance and stability in attenuating the disturbance and target forcing functions - are 
(a) visual gain

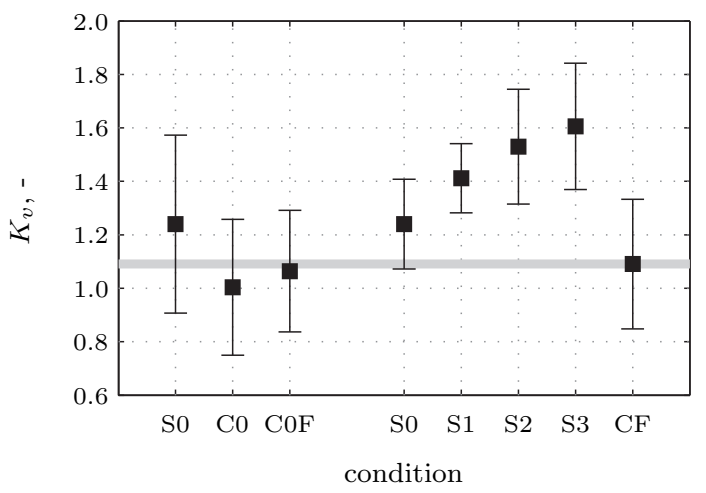

(c) visual lead

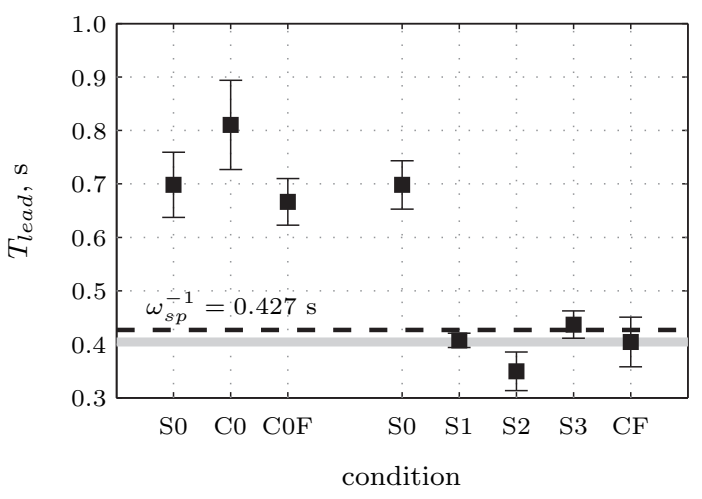

(e) visual time delay

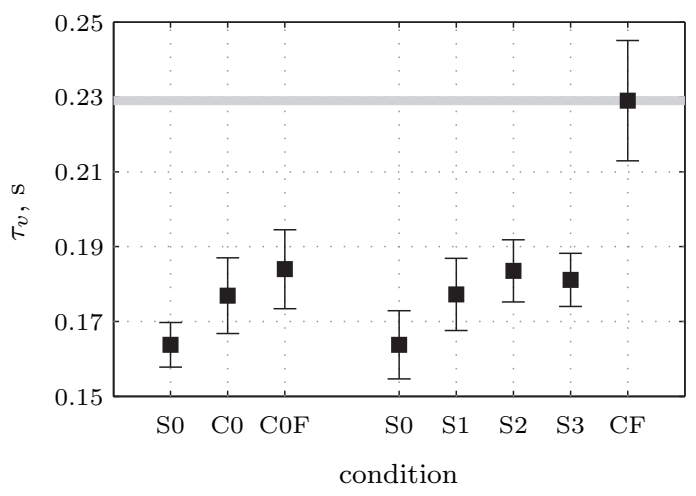

(g) neuromuscular damping

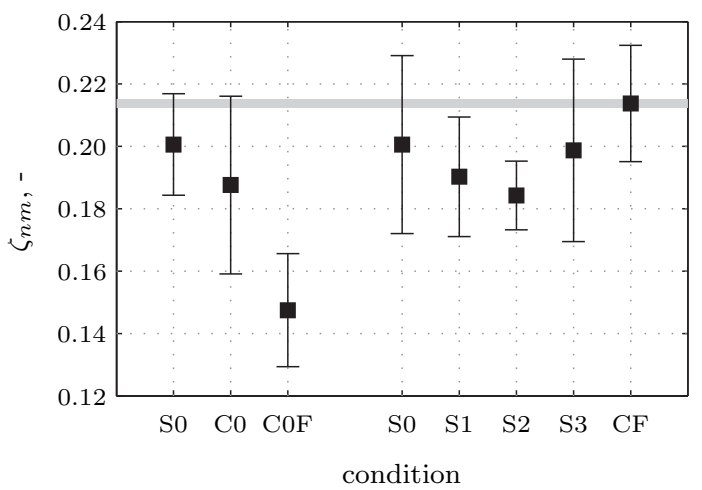

(b) vestibular gain

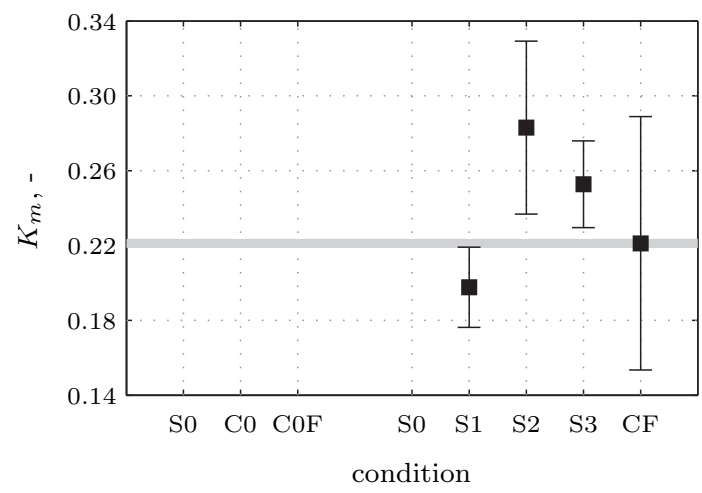

(d) visual lag

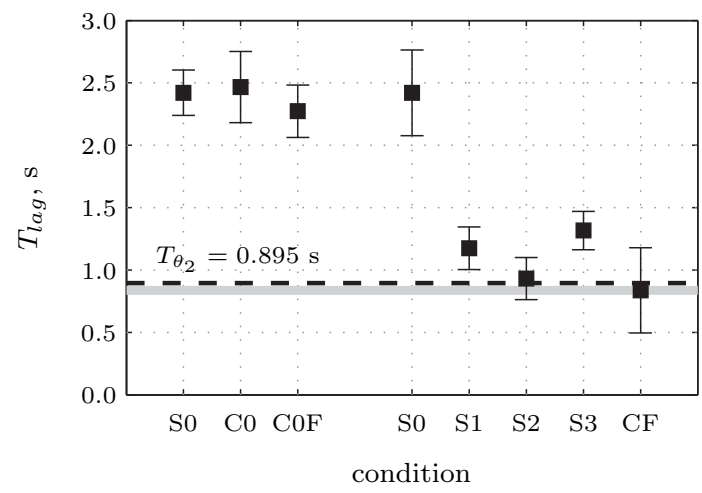

(f) vestibular time delay

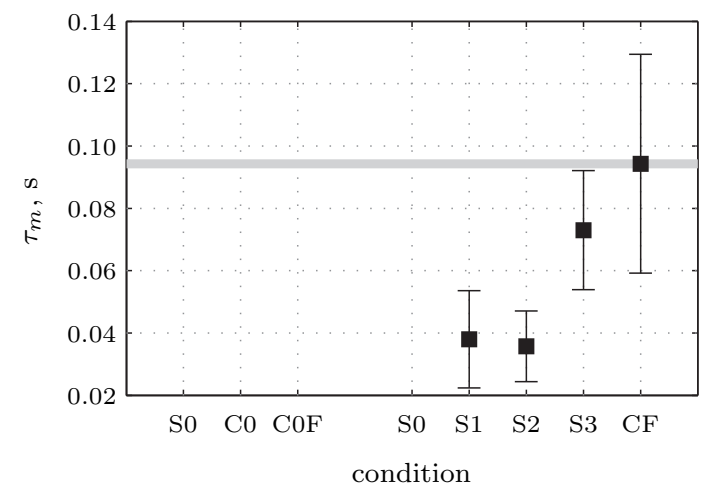

(h) neuromuscular frequency

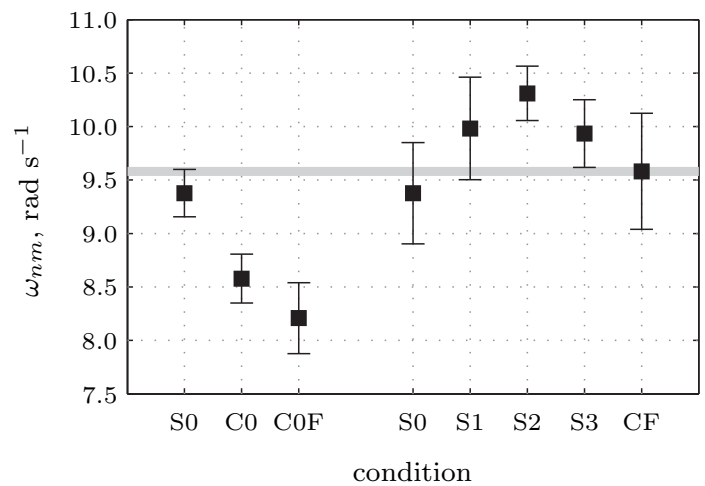

Figure 17. Multimodal pilot model parameters. 
(a) disturbance crossover frequency

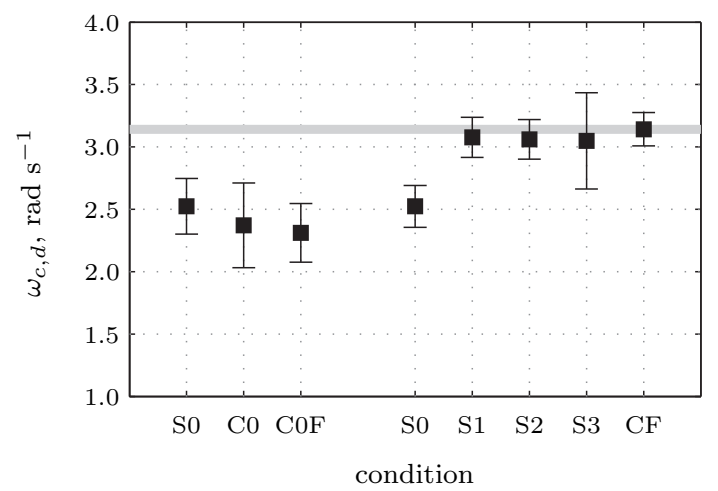

(c) disturbance phase margin

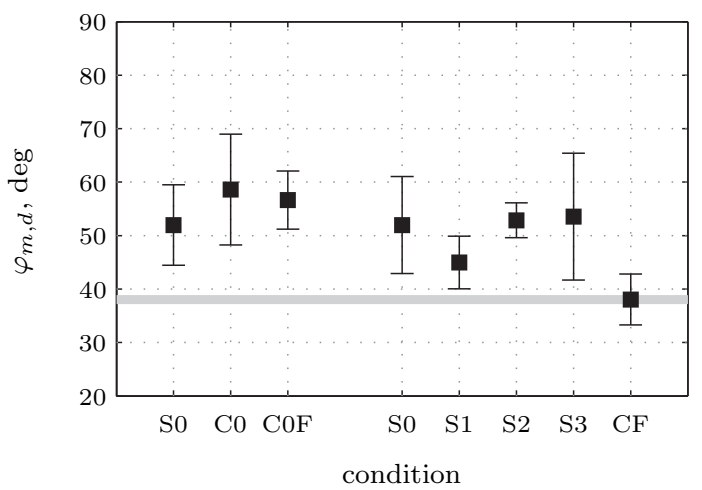

(b) target crossover frequency

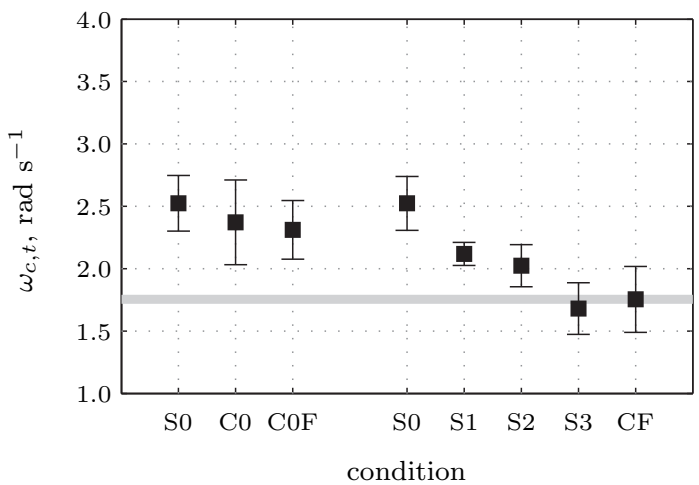

(d) target phase margin

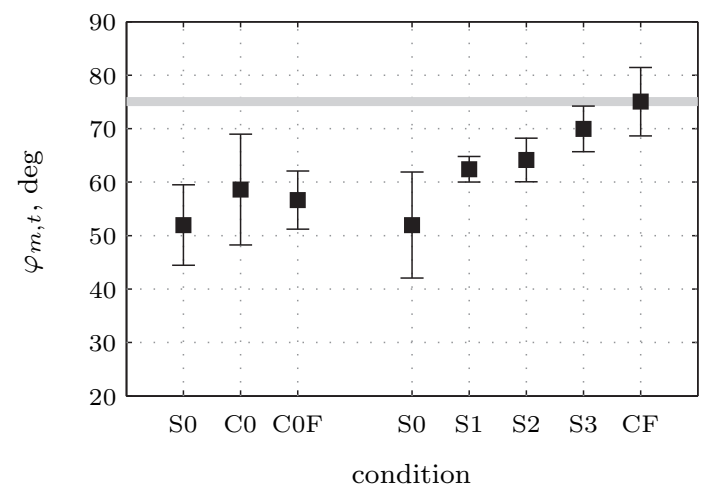

Figure 18. Crossover frequencies and phase margins.

Table 9. ANOVA results of crossover frequencies and phase margins, where $* *$ is highly significant $(p<0.01)$, * is significant $(0.01 \leq p<0.05)$, and - is not significant $(p \geq 0.05)$.

\begin{tabular}{|c|c|c|c|c|c|c|c|c|c|c|c|c|}
\hline \multirow{2}{*}{$\begin{array}{c}\text { Independent } \\
\text { variables }\end{array}$} & \multicolumn{12}{|c|}{ Dependent measures } \\
\hline & \multicolumn{3}{|c|}{$\omega_{c, d}$} & \multicolumn{3}{|c|}{$\omega_{c, t}$} & \multicolumn{3}{|c|}{$\varphi_{m, d}$} & \multicolumn{3}{|c|}{$\varphi_{m, t}$} \\
\hline Factor & $\mathrm{df}$ & $\mathrm{F}$ & Sig. & $\mathrm{df}$ & $\mathrm{F}$ & Sig. & $\mathrm{df}$ & $\mathrm{F}$ & Sig. & $\mathrm{df}$ & $\mathrm{F}$ & Sig. \\
\hline Environment (S0,C0,C0F) & 2,12 & 0.650 & - & 2,12 & 0.650 & - & 2,12 & 0.724 & - & 2,12 & 0.724 & - \\
\hline Motion (S0,S1,S2,S3,CF) & 4,24 & 6.211 & $* *$ & 4,24 & 13.710 & $* *$ & 4,24 & 3.790 & $*$ & $1.8,10.8$ & 10.069 & $* *$ \\
\hline
\end{tabular}

depicted in Figure 18. The corresponding ANOVA results are given in Table 9. Note that the disturbance and target open-loop responses, and thus disturbance and target crossover frequencies and phase margins, are equal for the conditions without motion $\left(H_{p \theta}=0\right)$ as can be derived from Equations 6 and 7 .

The disturbance and target crossover frequencies are depicted in Figures 18(a) and 18(b), respectively. The crossover frequencies were not affected by the experiment environment. The disturbance crossover frequency shows an increase for increasing motion fidelity, a significant effect. This effect is mainly present between the condition without motion S0 and the remaining motion conditions. A significant decrease in target crossover frequency can be observed for increasing motion fidelity. An increase in disturbance crossover frequency and decrease in target crossover frequency when adding pitch motion was also observed in previous experiments. ${ }^{4,5}$

Both the disturbance and target phase margins (Figures 18(c) and 18(d), respectively) are not significantly affected by experiment environment. The increase in motion fidelity yields a significant effect for the disturbance phase margin. For the increase in disturbance crossover frequency a slight decrease in corresponding disturbance phase margin can be observed. Consistent with the decrease in target crossover frequency shown in Figure 18(b) a significant increase in phase margin is visible in Figure 18(d). 


\section{Discussion}

Seven experienced pilots participated in an experiment where, for the first time, multimodal pilot pitch control behavior was compared between real flight and four different motion conditions in a motion-base flight simulator. The purpose of the experiment was to determine the effects of simulator motion fidelity on pilot performance and multimodal control behavior.

\section{V.A. Experiment Environment and Apparatus}

To isolate the effects of limited simulator motion, it is extremely important that all the remaining experimental variables - such as sidestick dynamics, display characteristics, and instructions to pilots - do not affect the comparison of measurements taken in the simulator and in the aircraft (Section II.F). Considerable effort was put into matching these experimental variables in the two apparatus as closely as possible. However, some differences were known to be present. Some examples of differences are that pilots were wearing a hood in the aircraft but not in the simulator, much more ambient light was present in the aircraft as compared to the simulator, and the display in the aircraft was slightly tilted compared to the display in the simulator (see Figures 7 and 10).

To investigate if these additional differences in experimental variables had an effect on performance and control behavior, three conditions were performed where pilots controlled the same aircraft dynamic model without any motion feedback. S0 was performed in the simulator, $\mathrm{C} 0$ in the aircraft on the ground, and $\mathrm{COF}$ in the aircraft in flight. The dependent measures from these three conditions were analyzed using an ANOVA to determine if the different experiment environment or apparatus introduced any significant effects. Despite our efforts to minimize differences between the experimental setups, significant effects were found in some of the dependent measures.

Pilot performance in the simulator was slightly better as compared to the aircraft. This was caused by a better target error reduction in the simulator. As the target error is directly visible on the display, this is most likely the result of better visibility of the target error on the simulator display. Differences in ambient light conditions, the slightly tilted screen in the simulator, or the difference in graphics processing hardware between the two setups could be the cause of this. The slightly higher pilot model visual time delay in the aircraft also indicates that more time was needed to process the information from the aircraft visual display. Note, however, that the latency in the display hardware was measured to be the same in both the aircraft and the simulator.

Finally, the pilot model neuromuscular damping ratio and natural frequency in the aircraft were significantly lower than in the simulator. Although the force-voltage characteristics of both sticks were exactly the same, small differences in other stick characteristics probably caused this significant effect. The sidestick in the simulator is an active stick that was set up to be completely fixed during the experiment. The force sidestick in the aircraft is a passive stick that moves slightly when force is applied. The significant effect in the neuromuscular parameters could also be introduced by the small difference in relative position of the pilot with respect to the sidestick in both apparatus.

Overall, more highly significant effects were found between the motion fidelity conditions. In addition, the effects found between conditions S3 and CF were generally a continuation of the trend for increasing motion fidelity in the simulator (S0-S3). Therefore, the observed effects in the dependent measures between the simulator conditions S0-S3 and the in-flight condition CF are expected to be mainly caused by the change in motion fidelity and are only slightly affected by the differences in experimental setup.

However, cross coupling between motion fidelity and other differences in experimental variables could also be present. For example, the hood pilots wore in the aircraft could have had no effect in the in-flight aircraft condition without motion, but could have had a significant effect in the aircraft in full motion due to its momentum. These cross-coupling effects can not be examined using the current experiment setup.

Based on pilot comments, there was no indication of a significant psychological effect of controlling the actual aircraft in flight as opposed to controlling in the relatively safe environment of a flight simulator. However, a significant smaller visual perception gain was observed for the in-flight full-motion condition, possibly indicating that pilots controlled with more caution in the real aircraft.

To eliminate fully the experimental effects other than the effects of motion fidelity, the entire experiment should be conducted in a single environment and apparatus. This requires a flight simulator with a very large motion space, to allow for the simulation of full unfiltered aircraft motion. Another possibility would be to utilize the experimental FBW system in the Citation II laboratory aircraft to simulate filtered aircraft 
motion in flight. However, without the possibility of direct lift control in the current aircraft configuration, pitch and heave motion would still be coupled.

\section{V.B. Motion Fidelity}

Pilot performance, determined by the total variance of the error, was significantly affected when motion fidelity was increased to full aircraft motion in the aircraft. A significant increase in performance was observed when pitch heave motion fidelity was increased, followed by a decrease in performance when c.g. heave was present. The decrease in overall tracking performance with c.g. heave shows that this heave component acts as a disturbance on the aircraft pitch rotational and pitch heave motion, decreasing the pilots' use of motion cues to improve performance. ${ }^{4}$ This indicates that adequate simulation of c.g. heave motion is required to not let pilots perform better in the simulator as compared to real flight.

An increase in disturbance-rejection performance for increasing motion fidelity was observed by a significant reduction of the disturbance variance component of the error. In addition, a significant increase in disturbance crossover frequency and a reduction in the disturbance phase margin were observed for improved motion fidelity. This reveals that pilots were able to compensate better for the disturbance as motion fidelity was increased.

Multimodal pilot control behavior was significantly affected by the level of motion fidelity. The most significant effects were found for the visual lead and lag time constants, and the visual and motion perception time delays. Visual lead significantly decreased when the level of pitch heave motion fidelity was increased. In addition, a small increase in visual lead can be observed when c.g. heave motion is present. This complies with previous research and again shows that c.g. heave reduces the pilots' use of physical motion cues in exchange for visual lead. ${ }^{4}$ A significant decrease in vestibular VAF for conditions with c.g. heave motion complements this finding. The lag time constant, which is strongly coupled with the lead time constant, shows similar effects. For the conditions with motion, S1-CF, the lead and lag time constants approximate the characteristic time constants of the aircraft pitch dynamics, indicating that pilots can adjust to the controlled dynamics much more accurately when motion is present.

The visual perception time delay increased significantly with increasing motion fidelity. A remarkable result is the significantly higher visual time delay for the in-flight full-motion condition, as compared to the simulator conditions. Differences between the display in the aircraft and the simulator might have contributed to this effect. However, as the differences in visual time delays between the no-motion conditions performed in the aircraft and the simulator are much smaller, this effect is most likely also caused by the level of motion fidelity. Finally, the vestibular time delay shows a significant increase when c.g. heave motion is present.

An increase in perceptual time delays for higher simulator motion fidelity has also been observed in previous experiments where a combined target-following disturbance-rejection task was used. ${ }^{4,5}$ In this type of task, the visual and physical motion cues provide conflicting information (see Figure 2). As motion fidelity increases, the conflict between the two cues increases as well, resulting in an increase in perceptual time delays, as more time is needed to process the information.

When subjective and objective measures are compared, the same overall trend between no-motion and motion conditions can be observed. For example, the rating between the simulator no-motion and motion conditions increased, while the pilot model lead and lag time constants decreased. However, the objective measures are clearly affected between the different simulator motion conditions, whereas pilots rated these conditions equally. Although, to gain pilot acceptance, subjective comments and ratings should still be an important part of the motion filter tuning process, this indicates that the presented objective measures have more potential to determine simulator motion fidelity.

Based on the four motion conditions evaluated in the current experiment, the condition with full pitch rotational motion and filtered pitch and c.g. heave (S3) induces performance levels and pilot control behavior most comparable to the baseline full-motion condition in the aircraft. However, more research is needed to determine a set of optimal simulator motion cueing settings that induce multimodal pilot control behavior that best approximates in-flight pilot control behavior.

Finally, it should be noted that aircraft size is an important factor in this discussion. This experiment was conducted in a Cessna Citation II, which is relatively small. In large commercial airliners, the pilot position is much further in front of the c.g., increasing the relative strength of the pitch heave component. This most likely changes how pilot control behavior is affected by different levels of heave motion fidelity and suggests that the results presented here cannot be directly extended to larger aircraft. More research is required. 


\section{Conclusions}

Pilots performed a pitch target-following disturbance-rejection task in a simulator under different motion cueing settings, in addition to performing the task in an aircraft in flight, the baseline condition. Except for the applied variation in motion fidelity, differences in experimental setup between the aircraft and the simulator were kept as small as possible. Pilot performance and control behavior were slightly affected by differences in the display and sidestick setup. However, the effects introduced by motion fidelity were far more apparent. For improved motion fidelity up to and including the full-motion condition in the aircraft, pilot disturbance-rejection performance significantly improved as indicated by the decrease in disturbance error variance and increase in disturbance crossover frequency. This improvement in performance was possible due to a significant change in multimodal pilot control behavior. For higher levels of motion fidelity, the visual lead time constant decreased, while visual and vestibular time delays increased. Multimodal pilot control behavior in the simulator motion condition with full pitch motion and filtered pitch and c.g. heave motion best approximates in-flight pilot control behavior.

\section{Acknowledgments}

The authors would like to thank all the members of the Flight Department of the TU Delft Faculty of Aerospace Engineering for their help in preparing the in-flight experiments. We would also like to thank the Netherlands' National Aerospace Laboratory (NLR) for their support in preparing and conducting the experiments. Finally, we would like to express our gratitude to the pilots who participated in the experiment.

This project was supported by the Technology Foundation STW, the applied science division of NWO, and the technology program of the Ministry of Economic Affairs.

\section{References}

${ }^{1}$ Feddersen, W. E., "The Role of Motion Information and its Contribution to Simulation Validity," Tech. Rep. D228-429001, Bell Helicopter Company, April 1962.

${ }^{2}$ Jex, H. R., Magdaleno, R. E., and Junker, A. M., "Roll Tracking Effects of G-vector Tilt and Various Types of Motion Washout," Fourteenth Annual Conference on Manual Control, University of Southern California, Los Angeles (CA), April 25-27 1978, pp. 463-502.

${ }^{3}$ Stapleford, R. L., Peters, R. A., and Alex, F. R., "Experiments and a Model for Pilot Dynamics with Visual and Motion Inputs," NASA Contractor Report NASA CR-1325, NASA, 1969.

${ }^{4}$ Zaal, P. M. T., Pool, D. M., de Bruin, J., Mulder, M., and van Paassen, M. M., "Use of Pitch and Heave Motion Cues in a Pitch Control Task," Journal of Guidance, Control, and Dynamics, Vol. 32, No. 2, March-April 2009, pp. 366-377.

${ }^{5}$ Pool, D. M., Zaal, P. M. T., van Paassen, M. M., and Mulder, M., "Effects of Heave Washout Settings in Aircraft Pitch Disturbance Rejection," Journal of Guidance, Control, and Dynamics, Vol. 33, No. 1, Jan.-Feb. 2010, pp. $29-41$.

${ }^{6}$ Hess, R. A. and Marchesi, F., "Analytical Assessment of Flight Simulator Fidelity Using Pilot Models," Journal of Guidance, Control, and Dynamics, Vol. 32, No. 3, May-June 2009, pp. 760-770.

${ }^{7}$ van den Berg, P., Zaal, P. M. T., Mulder, M., and van Paassen, M. M., "Conducting multi-modal pilot model identification - Results of a simulator experiment," Proceedings of the AIAA Modeling and Simulation Technologies Conference and Exhibit, Hilton Head (SC), No. AIAA-2007-6892, 20-23 Aug. 2007.

${ }^{8}$ Mooij, H. A., "In-Flight Measured Human Pilot Describing Function and Remnant for Pitch Attitude Control," Proceedings of the Ninth Annual Conference on Manual Control, Massachusetts Institute of Technology, Cambridge (MA), May 23-25 1973, pp. 311-318.

${ }^{9}$ van Gool, M. F. C. and Mooij, H. A., "A Comparison of In-Flight and Ground-Based Pitch Attitude Tracking Experiments," Proceedings of the Twelfth Annual Conference on Manual Control, University of Illinois, Urbana (IL), May 25-27 1976, pp. 443-454.

${ }^{10}$ Newell, F. D. and Pietrzak, P. E., "In-Flight Measurement of Human Response Characteristics," Journal of Aircraft, Vol. 5, No. 3, May-June 1968, pp. 277-284.

${ }^{11}$ Newell, F. D. and Smith, H. J., "Human Transfer Characteristics in Flight and Ground Simulation for a Roll Tracking Task," Tech. Rep. NASA TN D-5007, NASA, 1969.

${ }^{12}$ Hess, R. A. and Mnich, M. A., "Identification of Pilot-Vehicle Dynamics from In-Flight Tracking Data," Journal of Guidance, Control, and Dynamics, Vol. 9, No. 4, July-Aug. 1986, pp. 433-440.

${ }^{13}$ Steurs, M., Mulder, M., and van Paassen, M. M., "A Cybernetic Approach to Assess Flight Simulator Fidelity," Proceedings of the AIAA Modelling and Simulation Technologies Conference and Exhibit, Providence (RI), No. AIAA-2004-5442, 16-19 Aug. 2004.

${ }^{14}$ Ferguson, S. W., Clement, W. F., Cleveland, W. B., and Key, D. L., "Assessment of Simulation Fidelity Using Measurements of Piloting Technique in Flight," Proceedings of the 40th American Helicopter Society Annual Forum, Arlington (VA), May 16-18 1984. 
${ }^{15}$ Nieuwenhuizen, F. M., Zaal, P. M. T., Mulder, M., van Paassen, M. M., and Mulder, J. A., "Modeling Human Multichannel Perception and Control Using Linear Time-Invariant Models," Journal of Guidance, Control, and Dynamics, Vol. 31, No. 4, July-Aug. 2008, pp. 999-1013.

${ }^{16}$ Zaal, P. M. T., Pool, D. M., Mulder, M., and van Paassen, M. M., "Multimodal Pilot Control Behavior in Combined Target-Following Disturbance-Rejection Tasks," Journal of Guidance, Control, and Dynamics, Vol. 32, No. 5, Sept.-Oct. 2009, pp. $1418-1428$.

${ }^{17}$ Zaal, P. M. T., Pool, D. M., Postema, F. N., in 't Veld, A. C., Mulder, M., van Paassen, M. M., and Mulder, J. A., "Design and Certification of a Fly-By-Wire System with Minimal Impact on the Original Flight Controls," Proceedings of the AIAA Guidance, Navigation, and Control Conference and Exhibit, Chicago (IL), No. AIAA-2009-5985, 10-13 Aug. 2009.

${ }^{18}$ McRuer, D. T. and Jex, H. R., "A Review of Quasi-Linear Pilot Models," IEEE Transactions on Human Factors in Electronics, Vol. HFE-8, No. 3, 1967, pp. 231-249.

${ }^{19}$ Schmidt, S. F. and Conrad, B., "Motion Drive Signals for Piloted Flight Simulators," Tech. Rep. NASA CR-1601, Ames Research Center, National Aeronautics and Space Administration, 1970.

${ }^{20}$ Kaljouw, W. J., Mulder, M., and van Paassen, M. M., "Multi-loop Identification of Pilot's Use of Central and Peripheral Visual Cues," Proceedings of the AIAA Modelling and Simulation Technologies Conference and Exhibit, Providence (RI), No. AIAA-2004-5443, 16-19 Aug. 2004.

${ }^{21}$ van Paassen, M. M. and Mulder, M., "Identification of Human Operator Control Behaviour in Multiple-Loop Tracking Tasks," Proceedings of the Seventh IFAC/IFIP/IFORS/IEA Symposium on Analysis, Design and Evaluation of Man-Machine Systems, Kyoto Japan, Pergamon, Kidlington, Sept. 16-18 1998, pp. 515-520.

${ }^{22}$ Zaal, P. M. T., Pool, D. M., Chu, Q. P., van Paassen, M. M., Mulder, M., and Mulder, J. A., "Modeling Human Multimodal Perception and Control Using Genetic Maximum Likelihood Estimation," Journal of Guidance, Control, and Dynamics, Vol. 32, No. 4, July-Aug. 2009, pp. 1089-1099.

${ }^{23}$ Nieuwenhuizen, F. M., van Paassen, M. M., Mulder, M., and Bülthoff, H. H., "Implementation and Validation of a Model of the MPI Stewart Platform," Proceedings of the AIAA Guidance, Navigation, and Control Conference and Exhibit, Toronto (ON), Canada, No. AIAA-2010-8217, 2-5 Aug. 2010.

${ }^{24}$ Hosman, R. J. A. W., Pilot's Perception and Control of Aircraft Motions, Doctoral dissertation, Faculty of Aerospace Engineering, Delft University of Technology, 1996, http://repository.tudelft.nl.

${ }^{25}$ van der Vaart, J. C., Modelling of Perception and Action in Compensatory Manual Control Tasks, Doctoral dissertation, Faculty of Aerospace Engineering, Delft University of Technology, 1992, http://repository.tudelft.nl.

${ }^{26}$ Mulder, M., Lubbers, B., Zaal, P. M. T., van Paassen, M. M., and Mulder, J. A., "Aerodynamic Hinge Moment Coefficient Estimation Using Automatic Fly-By-Wire Control Inputs," Proceedings of the AIAA Modeling and Simulation Technologies Conference and Exhibit, Chicago (IL), No. AIAA-2009-5692, 10-13 Aug. 2009.

${ }^{27}$ Zaal, P. M. T., Pool, D. M., Mulder, M., van Paassen, M. M., and Mulder, J. A., "Identification of Multimodal Pilot Control Behavior in Real Flight," Journal of Guidance, Control, and Dynamics, Vol. 33, No. 5, Sept.-Oct. 2010, pp. $1527-1538$.

${ }^{28}$ McRuer, D. T., Ashkenas, I., and Graham, D., Aircraft Dynamics and Automatic Control, Princeton University Press, Princeton, New Jersey, 1973.

${ }^{29}$ Damveld, H. J., Beerens, G. C., van Paassen, M. M., and Mulder, M., "Design of Forcing Functions for the Identification of Human Control Behavior," Journal of Guidance, Control, and Dynamics, Vol. 33, No. 4, July - Aug. 2010, pp. $1064-1081$.

${ }^{30}$ McRuer, D. T., Graham, D., Krendel, E. S., and Reisener, W., "Human Pilot Dynamics in Compensatory Systems. Theory, Models and Experiments With Controlled Element and Forcing Function Variations," Tech. Rep. AFFDL-TR-65-15, Wright Patterson AFB (OH): Air Force Flight Dynamics Laboratory, 1965.

${ }^{31}$ Hosman, R. J. A. W., Grant, P., and Schroeder, J. A., "Pre and Post Pilot Model Analyis Compared to Experimental Simulator Results," AIAA Modeling and Simulation Technologies Conference and Exhibit, San Francisco (CA), No. AIAA2005-6303, 15-18 Aug. 2005.

${ }^{32}$ Pool, D. M., Zaal, P. M. T., Damveld, H. J., van Paassen, M. M., van der Vaart, J. C., and Mulder, M., "Pilot Equalization in Manual Control of Aircraft Pitch Dynamics," Journal of Guidance, Control, and Dynamics, submitted for publication December 2010.

${ }^{33}$ Berkouwer, W. R., Stroosma, O., van Paassen, M. M., Mulder, M., and Mulder, J. A., "Measuring the Performance of the SIMONA Research Simulator's Motion System," Proceedings of the AIAA Modeling and Simulation Technologies Conference and Exhibit, San Francisco (CA), No. AIAA-2005-6504, 15-18 Aug. 2005.

${ }^{34}$ Stroosma, O., van Paassen, M. M., Mulder, M., and Postema, F. N., "Measuring Time Delays in Simulator Displays," Proceedings of the AIAA Modeling and Simulation Technologies Conference and Exhibit, Hilton Head (SC), No. AIAA-20076562, 20-23 Aug. 2007.

${ }^{35}$ Beukers, J. T., Stroosma, O., Pool, D. M., Mulder, M., and van Paassen, M. M., "Investigation into Pilot Perception and Control During Decrab Maneuvers in Simulated Flight," Journal of Guidance, Control, and Dynamics, Vol. 33, No. 4, 2010, pp. 1048-1063.

${ }^{36}$ Schroeder, J. A. and Grant, P. R., "Pilot Behavioral Observations in Motion Flight Simulation," Proceedings of the AIAA Guidance, Navigation, and Control Conference and Exhibit, Toronto (ON), Canada, No. AIAA-2010-8353, 2-5 Aug. 2010.

${ }^{37}$ Field, A., Discovering Statistics Using SPSS, ISM Introducing Statistical Methods, SAGE Publications Ltd., 1 Oliver's Yard, 55 City Road, London EC1Y 1SP, 2nd ed., 2005. 